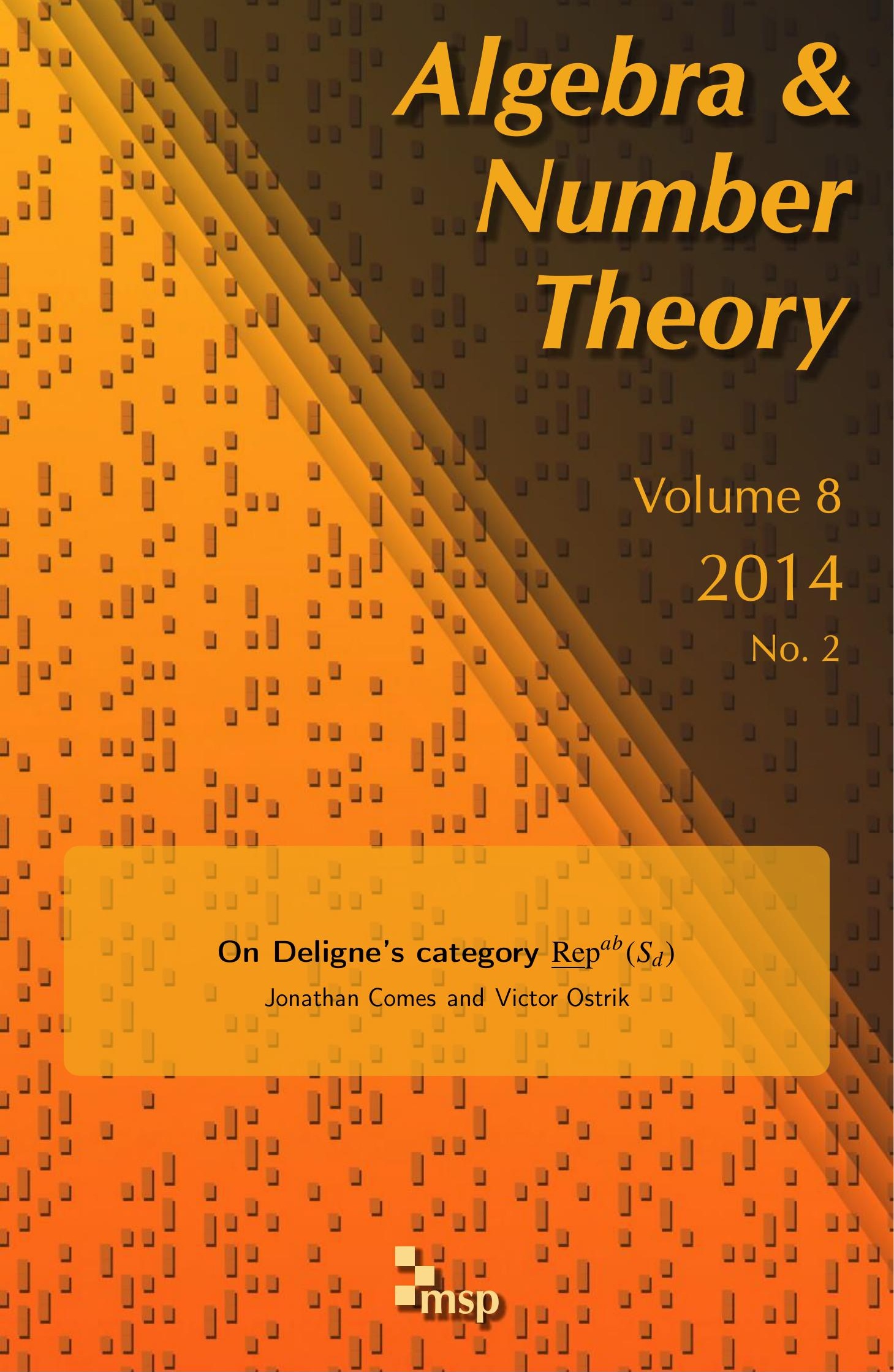




\title{
On Deligne's category $\underline{\operatorname{Rep}}^{a b}\left(S_{d}\right)$
}

\author{
Jonathan Comes and Victor Ostrik
}

Dedicated to the memory of Andrei Zelevinsky

We prove a universal property of Deligne's category $\underline{\operatorname{Rep}}^{a b}\left(S_{d}\right)$. Along the way, we classify tensor ideals in the category $\underline{\operatorname{Rep}}\left(S_{d}\right)$.

\section{Introduction}

1A. Let $F$ be a field of characteristic zero and let $I$ be a finite set. Let $S_{I}$ be the symmetric group of the permutations of $I$ and let $\operatorname{Rep}\left(S_{I}\right)$ be the category of finite-dimensional $F$-linear representations of $S_{I}$ considered as a symmetric tensor category. Let $X_{I} \in \operatorname{Rep}\left(S_{I}\right)$ be the space of $F$-valued functions on $I$ with an obvious action of $S_{I}$. The object $X_{I}$ with pointwise operations has a natural structure of associative commutative algebra with unit $1_{X_{I}}$ in the category $\operatorname{Rep}\left(S_{I}\right)$. We have a morphism $\operatorname{Tr}: X_{I} \rightarrow F$ defined as a trace of the operator of left multiplication; clearly the map $X_{I} \otimes X_{I} \rightarrow F$ given by $x \otimes y \mapsto \operatorname{Tr}(x y)$ is a nondegenerate pairing. Finally, $\operatorname{Tr}\left(1_{X_{I}}\right)=\operatorname{dim}\left(X_{I}\right)=|I|$ where $|I| \geq 0$ is the cardinality of $I$.

Now let $G$ be a finite group acting on $d$-dimensional associative commutative unital algebra $T$ over $F$ such that the pairing $\operatorname{Tr}(x y)$ is nondegenerate. It is easy to see $^{1}$ that there exists a finite set $I$ with $|I|=d$ and an essentially unique tensor functor $F: \operatorname{Rep}\left(S_{I}\right) \rightarrow \operatorname{Rep}(G)$ such that $F\left(X_{I}\right) \simeq T$ (isomorphism of $G$-algebras); in this sense the category $\operatorname{Rep}\left(S_{I}\right)$ is a universal category (in the realm of representation categories of finite groups) with object $X_{I}$ as above.

1B. For an arbitrary symmetric tensor category $\mathscr{T}$ one can consider objects $T \in \mathcal{T}$ satisfying the following:

(a) $T$ has a structure of associative commutative algebra (given by the multiplication map $\mu_{T}: T \otimes T \rightarrow T$ ) with unit (given by the map $1_{T}: \mathbf{1} \rightarrow T$ ).

MSC2010: primary 18D10; secondary 19D23.

Keywords: tensor categories, symmetric group.

${ }^{1}$ Set $I$ to be the set of $F$-algebra homomorphisms $T \rightarrow \bar{F}$ where $\bar{F}$ is an algebraic closure of $F$ and use an obvious homomorphism $G \rightarrow S_{I}$. 
(b) The object $T$ is rigid. Moreover, if we define the map $\operatorname{Tr}: T \rightarrow \mathbf{1}$ as the composition

$$
T \stackrel{\mathrm{id}_{T} \otimes \operatorname{coev}_{T}}{\longrightarrow} T \otimes T \otimes T^{*} \stackrel{\mu_{T} \otimes \mathrm{id}_{T^{*}}}{\longrightarrow} T \otimes T^{*} \simeq T^{*} \otimes T \stackrel{\mathrm{ev}_{T}}{\longrightarrow} \mathbf{1},
$$

then the pairing $T \otimes T \stackrel{\mu_{T}}{\rightarrow} T \stackrel{\operatorname{Tr}}{\rightarrow} \mathbf{1}$ is nondegenerate, that is, it corresponds to an isomorphism $T \simeq T^{*}$ under the identification $\operatorname{Hom}(T \otimes T, \mathbf{1})=\operatorname{Hom}\left(T, T^{*}\right)$.

(c) We have $\operatorname{dim}(T)=t \in F$ (equivalently, $\operatorname{Tr}\left(1_{T}\right)=t$ ).

For an arbitrary $t \in F$, Deligne [2007] defined a symmetric tensor category $\underline{\operatorname{Rep}}\left(S_{t}\right)$ with a distinguished object $X$ which is universal in the following sense:

Proposition 1.1 [Deligne 2007, Proposition 8.3]. Let $\mathscr{T}$ be a Karoubian symmetric tensor category over $F$. The functor $\mathscr{F}_{F} \mapsto \mathscr{F}(X)$ is an equivalence of the category of braided tensor functors $\operatorname{Rep}\left(S_{t}\right) \rightarrow \mathcal{T}$ with the category of objects $T \in \mathcal{T}$ satisfying (a), (b), (c) above and their isomorphisms.

Note that for $t=d \in \mathbb{Z}_{\geq 0}$, Proposition 1.1 applied to $T=X_{I}$ (with $|I|=d$ ) produces a canonical functor $\underline{\operatorname{Rep}}\left(S_{d}\right) \rightarrow \operatorname{Rep}\left(S_{d}\right)$ (where $S_{d}:=S_{I}$ ). It is known (see [Deligne 2007, Théorème 6.2]) that this functor is surjective on Hom's. Moreover, the morphisms sent to zero by this functor are precisely the so-called negligible morphisms (see [Deligne 2007, §6.1]).

1C. The category $\underline{\operatorname{Rep}}\left(S_{t}\right)$ is a Karoubian category; it is not abelian for $t=d \in \mathbb{Z}_{\geq 0}$. Remarkably, in [2007, Proposition 8.19] Deligne defined an abelian symmetric tensor category $\underline{\operatorname{Rep}}^{a b}\left(S_{d}\right)$ and a fully faithful braided tensor functor $\underline{\operatorname{Rep}}\left(S_{d}\right) \rightarrow$ $\operatorname{Rep}^{a b}\left(S_{d}\right){ }^{2}$ The main goal of this paper is to prove a certain universal property of the category $\operatorname{Rep}^{a b}\left(S_{d}\right)$ conjectured in [Deligne 2007, Conjecture 8.21].

To state this property we need to use the language of algebraic geometry within an abelian symmetric tensor category $\mathscr{T}$ (see [Deligne 1990]). Namely, for an object $T \in \mathscr{T}$ satisfying (a), (b), (c) above we can talk about the (affine) $\mathscr{T}$-scheme I $:=\operatorname{Spec}(T)$ and the affine group scheme $S_{\mathbf{I}}$ of its automorphisms; see [Deligne 2007, §8.10]. Furthermore, assume that the category $\mathcal{T}$ is pre-Tannakian (see Section 2A below), that is, it satisfies the finiteness conditions in [Deligne 1990, 2.12.1]. Recall that in this case a fundamental group of $\mathscr{T}$ is defined in [Deligne 1990, $\S 8.13]$. This is an affine group scheme $\pi \in \mathscr{T}$ which acts functorially on any object of $\mathcal{T}$ and this action is compatible with a formation of tensor products. In particular, the action of $\pi$ on $T$ gives a homomorphism $\varepsilon: \pi \rightarrow S_{\mathbf{I}}$. Let $\operatorname{Rep}\left(S_{\mathbf{I}}\right)$ be the category of representations of $S_{\mathbf{I}}$ (see [Deligne 2007, §8.10]) and let $\operatorname{Rep}\left(S_{\mathbf{I}}, \varepsilon\right)$ be the full subcategory of $\operatorname{Rep}\left(S_{\mathbf{I}}\right)$ consisting of such representations $\rho: S_{\mathbf{I}} \rightarrow \operatorname{GL}(V)$ that the action $\rho \circ \varepsilon$ of $\pi$ on $V$ coincides with the canonical action (see [Deligne 2007,

\footnotetext{
${ }^{2}$ We refer the reader to [Deligne 2007, §5.8] for an example of Karoubian symmetric tensor category which admits no braided tensor functor to an abelian symmetric tensor category.
} 
$\S 8.20]) . \operatorname{Rep}\left(S_{\mathbf{I}}, \varepsilon\right)$ is an abelian symmetric tensor category and $T$ is one of its objects. It follows that the functor $\mathscr{F}: \underline{\operatorname{Rep}}\left(S_{t}\right) \rightarrow \mathscr{T}$ constructed in Proposition 1.1 factorizes as $\underline{\operatorname{Rep}}\left(S_{t}\right) \stackrel{\mathscr{F}_{T} T}{\rightarrow} \operatorname{Rep}\left(S_{\mathbf{I}}, \varepsilon\right) \rightarrow \widetilde{\mathcal{T}}$, where the functor $\mathscr{F}_{T}$ is constructed by applying Proposition 1.1 to $T \in \operatorname{Rep}\left(S_{\mathbf{I}}, \varepsilon\right)$ and $\operatorname{Rep}\left(S_{\mathbf{I}}, \varepsilon\right) \rightarrow \mathscr{T}$ is the forgetful functor. Here is the main result of this paper:

Theorem 1.2 (compare [Deligne 2007, 8.21.2]). Let $\mathscr{T}$ be a pre-Tannakian category and $T \in \mathcal{T}$ be an object satisfying (a), (b), (c) from Section $1 B$ with $t=d \in \mathbb{Z}_{\geq 0} \subset F$. Then the category $\operatorname{Rep}\left(S_{\mathbf{I}}, \varepsilon\right)$ endowed with the functor $\mathscr{F}_{T}: \underline{\operatorname{Rep}}\left(S_{d}\right) \rightarrow \operatorname{Rep}\left(S_{\mathbf{I}}, \varepsilon\right)$ is equivalent to one of the following:

(a) $\operatorname{Rep}\left(S_{d}\right)$ together with the functor $\underline{\operatorname{Rep}}\left(S_{d}\right) \rightarrow \operatorname{Rep}\left(S_{d}\right)$ from Section $1 B$.

(b) $\underline{\operatorname{Rep}}^{a b}\left(S_{d}\right)$ together with the fully faithful functor $\underline{\operatorname{Rep}}\left(S_{d}\right) \rightarrow \underline{\operatorname{Rep}}^{a b}\left(S_{d}\right)$ above.

Remark 1.3. A similar (and easier) statement holds true for $t \notin \mathbb{Z}_{\geq 0}$; see [Deligne 2007, Corollary B2].

1D. The forgetful functor $\operatorname{Rep}\left(S_{\mathbf{I}}, \varepsilon\right) \rightarrow \mathscr{T}$ above is an exact braided tensor functor. Thus Theorem 1.2 implies that for a pre-Tannakian category $\mathscr{T}$ a braided tensor functor $\mathscr{F}_{F}: \underline{\operatorname{Rep}}\left(S_{d}\right) \rightarrow \mathscr{T}$ either factorizes through $\underline{\operatorname{Rep}}\left(S_{d}\right) \rightarrow \operatorname{Rep}\left(S_{d}\right)$ or extends to an exact tensor functor $\underline{\operatorname{Rep}}^{a b}\left(S_{d}\right) \rightarrow \mathscr{T}$. A crucial step in our proof of Theorem 1.2 is a construction of the pre-Tannakian category $\mathscr{K}_{d}^{0}$ and fully faithful embedding $\underline{\operatorname{Rep}}\left(S_{d}\right) \subset \mathscr{K}_{d}^{0}$ such that we have the following extension property: a tensor (not necessarily braided) functor $\underline{\operatorname{Rep}}\left(S_{d}\right) \rightarrow \mathcal{T}$ either factorizes through $\underline{\operatorname{Rep}}\left(S_{d}\right) \rightarrow \operatorname{Rep}\left(S_{d}\right)$ or extends to an exact tensor functor $\mathcal{K}_{d}^{0} \rightarrow \mathscr{T}$; see Section 5A. Then we use general properties of the fundamental groups from [Deligne 1990, §8] in order to prove that $\mathscr{K}_{d}^{0}$ satisfies the universal property as in Theorem 1.2 and, in fact, is equivalent to $\underline{\operatorname{Rep}}^{a b}\left(S_{d}\right)$.

The following analogy plays a significant role in the proof of Theorem 1.2. Let $T L(q)$ be the Temperley-Lieb category; see, for example, [Freedman 2003, §A1]. Assume that $q$ is a nontrivial root of unity. It is well known that the category $T L(q)$ is tensor equivalent to the category of tilting modules over quantum SL(2); see, for example, [Ostrik 2008, proof of Theorem 2.4]. Thus $T L(q)$ is a Karoubian tensor category (braided but not symmetric) endowed with a fully faithful functor to the abelian tensor category $\mathscr{C}_{q}$ of finite-dimensional representations of quantum SL(2). On the other hand there exists a well known semisimple tensor category $\overline{\mathscr{C}}_{q}$ and a full tensor functor $T L(q) \rightarrow \overline{\mathscr{C}}_{q}$; see, e.g., [Andersen 1992, §4]. We consider the diagram $\overline{\mathscr{C}}_{q} \nleftarrow T L(q) \subset \mathscr{C}_{q}$ as a counterpart of the diagram $\operatorname{Rep}\left(S_{d}\right) \nleftarrow \underline{\operatorname{Rep}}\left(S_{d}\right) \subset \underline{\operatorname{Rep}}^{a b}\left(S_{d}\right)$.

The main technical result of [Ostrik 2008] states that tensor functors $T L(q) \rightarrow \mathscr{D}$ to certain abelian tensor categories $\mathscr{D}$ factorize either through $T L(q) \rightarrow \overline{\mathscr{C}}_{q}$ or through $T L(q) \subset \mathscr{C}_{q}$ (see [Ostrik 2008, §2.6]) which is reminiscent of the extension property of the category $\mathscr{K}_{d}^{0}$ above; see also [Ostrik 2008, Remark 2.10]. Thus 
in the construction of $\mathscr{K}_{d}^{0}$ we follow the strategy from [Ostrik 2008] with crucial use of information from [Comes and Ostrik 2011]. Namely, we find $\mathscr{K}_{d}^{0}$ inside the homotopy category of $\underline{\operatorname{Rep}}\left(S_{d}\right)$ as a heart of a suitable $t$-structure (see Section 4B). The definition of the $t$-structure is based on Lemma 3.11 (due to P. Deligne) and almost immediately implies the extension property of the category $\mathcal{K}_{d}^{0}$ mentioned above. However, the verification of the axioms of a $t$-structure is quite nontrivial. To do this we use a decomposition of the category $\operatorname{Rep}\left(S_{d}\right)$ into blocks described in [Comes and Ostrik 2011, Theorem 5.3]. We provide a blockwise description of the $t$-structure above in Section 4C2. We then observe that the description above coincides with the description of a well known $t$-structure on the blocks of the Temperley-Lieb category.

\section{Preliminaries}

2A. Tensor categories terminology. To us a tensor (or monoidal) category is a category with a tensor product functor endowed with an associativity constraint and a unit object 1; see, for example, [Bakalov and Kirillov 2001, Definition 1.1.7]. Recall that a tensor category is called rigid if any object admits both a left and right dual; see [ibid., Definition 2.1.1]. A braided tensor category is a tensor category equipped with a braiding; see [ibid., Definition 1.2.3]. A symmetric tensor category is a braided tensor category such that the square of the braiding is the identity.

Recall that $F$ is a fixed field of characteristic zero. All categories and functors considered in this paper are going to be $F$-linear. So, an $F$-linear tensor category (or tensor category over $F$ ) is a tensor category which is $F$-linear (but not necessarily additive) and such that the tensor product functor is $F$-bilinear. A Karoubian tensor category over $F$ is an $F$-linear tensor category which is Karoubian as an $F$-linear category (i.e., it is additive and every idempotent endomorphism is a projection to a direct summand). A tensor ideal $\mathscr{I}$ in a tensor category $\mathscr{T}$ consists of subspaces $\Phi(X, Y) \subset \operatorname{Hom}_{\mathscr{T}}(X, Y)$ for every $X, Y \in \mathscr{T}$ such that (i) $h \circ g \circ f \in \mathscr{I}(X, W)$ whenever $f \in \operatorname{Hom}_{\mathscr{T}}(X, Y), g \in \mathscr{I}(Y, Z), h \in \operatorname{Hom}_{\mathscr{T}}(Z, W)$, and (ii) $f \otimes \operatorname{id}_{Z} \in$ $\mathscr{I}(X \otimes Z, Y \otimes Z)$ whenever $f \in \mathscr{T}(X, Y)$. For example, if the category $\mathcal{T}$ has a well defined trace the collection of negligible morphisms ${ }^{3}$ forms a tensor ideal; see [Freedman 2003, §A1.3].

Finally we say that an $F$-linear symmetric tensor category $\mathscr{T}$ is pre-Tannakian if the following conditions are satisfied:

(a) All Hom's are finite-dimensional vector spaces over $F$ and $\operatorname{End}(\mathbf{1})=F$.

(b) $\mathcal{T}$ is an abelian category and all objects have finite length.

(c) $\mathcal{T}$ is rigid.

${ }^{3}$ Recall that a morphism $f \in \operatorname{Hom}_{\mathscr{T}}(X, Y)$ is negligible if $\operatorname{Tr}(f g)=0$ for any $g \in \operatorname{Hom}_{\mathscr{T}}(Y, X)$. We will call an object negligible if its identity morphism is negligible. 
Remark 2.1. In the terminology of [Deligne 1990] a pre-Tannakian category is the same as a "catégorie tensorielle" (see [ibid., §2.1]) satisfying a finiteness assumption [ibid., 2.12.1]. This is precisely the class of tensor categories over $F$ for which a fundamental group (see [ibid., §8]) is defined.

2B. The category $\operatorname{Rep}\left(S_{t}\right)$. We recall here briefly the construction of the category $\underline{\operatorname{Rep}}\left(S_{t}\right)$ following [Comes and Ostrik 2011, §2]. We refer the reader to loc. cit. and [Deligne 2007, §8] for much more detailed exposition.

2B1. The category $\operatorname{Rep}_{0}\left(S_{t}\right)$. Let $A$ be a finite set. A partition $\pi$ of $A$ is a collection of nonempty subsets $\pi_{i} \subset A$ such that $A=\bigsqcup_{i} \pi_{i}$ (disjoint union); the subsets $\pi_{i}$ are called parts of the partition $\pi$. We say that partition $\pi$ is finer than partition $\mu$ of the same set if any part of $\pi$ is a subset of some part of $\mu$. For three finite sets $A, B, C$ and the partitions $\pi$ of $A \sqcup B$ and $\mu$ of $B \sqcup C$ we define the partition $\mu \star \pi$ of $A \sqcup B \sqcup C$ as the finest partition such that parts of $\pi$ and $\mu$ are subsets of its parts. The partition $\mu \star \pi$ induces a partition $\mu \cdot \pi$ of $A \sqcup C$ such that parts of $\mu \cdot \pi$ are nonempty intersections of parts of $\mu \star \pi$ with $A \sqcup C \subset A \sqcup B \sqcup C$; we also define an integer $\ell(\mu, \pi)$ which is the number of parts of $\mu \star \pi$ contained in $B$.

Definition 2.2. Given $t \in F$, we define the $F$-linear symmetric tensor category $\underline{\operatorname{Rep}}_{0}\left(S_{t}\right)$ as follows:

- Objects: finite sets; object corresponding to a finite set $A$ is denoted $[A]$.

- Morphisms: $\operatorname{Hom}([A],[B])$ is the $F$-linear span of partitions of $A \sqcup B$; composition of morphisms represented by partitions $\pi \in \operatorname{Hom}([A],[B])$ and $\mu \in \operatorname{Hom}([B],[C])$ is $t^{\ell(\mu, \pi)} \mu \cdot \pi \in \operatorname{Hom}([A],[C])$.

- Tensor product: disjoint union (see [Comes and Ostrik 2011, Definition 2.15]); unit object is $[\varnothing]$; tensor product of morphisms, associativity and commutativity constraints are the obvious ones (see [ibid., §2.2]).

The category $\underline{\operatorname{Rep}}_{0}\left(S_{t}\right)$ has a distinguished object [pt] where pt is a one-element set. The object [pt] has a natural structure of commutative associative algebra in $\underline{\operatorname{Rep}}_{0}\left(S_{t}\right)$ where the multiplication (resp. unit) map is given by the partition of pt $\sqcup \mathrm{pt} \sqcup \mathrm{pt}$ (resp. pt) consisting of one part. It is immediate to check that the object [pt] satisfies conditions (a), (b), (c) from Section 1B. Moreover, we have the following universal property:

Proposition 2.3. Let $\mathcal{T}$ be an F-linear symmetric tensor category. The functor from the category of braided tensor functors $\mathscr{F}: \underline{\operatorname{Re}}_{0}\left(S_{t}\right) \rightarrow \mathscr{T}$ to the category of objects $T \in \mathcal{T}$ satisfying (a), (b), (c) from Section $1 B$ and their isomorphisms, which sends $\mathscr{F} \mapsto \mathscr{F}([\mathrm{pt}])$ and sends natural transformations $\left(\eta: \mathscr{F} \rightarrow \mathscr{F}^{\prime}\right) \mapsto \eta_{[\mathrm{pt}]}$, is an equivalence of categories. 
Sketch of proof. We restrict ourselves to a description of the inverse functor on objects; for more details, see [Deligne 2007, §8]. So assume that $T \in \mathcal{T}$ satisfies (a), (b), (c) from Section 1B. We define $\mathscr{F}([A])=T^{\otimes A}$ (here $T^{\otimes A}$ is a tensor product of copies of $T$ labeled by elements of $A$; since the category $\mathcal{T}$ is symmetric this is well defined). The tensor structure on the functor $\mathscr{F}$ will be given by the obvious isomorphisms $T^{\otimes A \sqcup B}=T^{\otimes A} \otimes T^{\otimes B}$. It remains to define $\mathscr{F}$ on the morphisms. Observe that a morphism from $\operatorname{Hom}([A],[B])$ represented by a partition $\pi$ of $A \sqcup B$ is a tensor product of morphisms corresponding to partitions with precisely one part $\pi=\bigotimes_{i} \pi_{i}$. Thus it is sufficient to define $\mathscr{F}(\pi)$ only for $\pi$ consisting of one part $A \sqcup B$. In this case we set $\mathscr{F}(\pi)=T^{\otimes A} \rightarrow T \rightarrow T^{\otimes B}$ where the first map is the multiplication morphism $T^{\otimes A} \rightarrow T$ and the second one is the dual to the multiplication morphism $T^{\otimes B} \rightarrow T$, where $T$ and $T^{*}$ are identified via (b) from Section 1B. One verifies that the assumptions (a), (b), (c) from Section 1B ensure that the tensor functor $\mathscr{F}$ is well defined.

2B2. The categories $\underline{\operatorname{Rep}}\left(S_{t}\right)$ and $\underline{\operatorname{Rep}}^{a b}\left(S_{d}\right)$.

Definition 2.4 (compare [Deligne 2007, Définition 2.17] or [Comes and Ostrik 2011, Definition 2.19]). The category $\operatorname{Rep}\left(S_{t}\right)$ is the Karoubian (or pseudoabelian) envelope $^{4}$ of the category $\underline{\operatorname{Rep}}_{0}\left(S_{t}\right)$.

It follows immediately from Proposition 2.3 that the category $\operatorname{Rep}\left(S_{t}\right)$ has universal property from Proposition 1.1. We now use this universal property to construct Deligne's category $\operatorname{Rep}^{a b}\left(S_{d}\right)$ from the introduction.

It is known (see [Deligne 2007, Théorème 2.18] or [Comes and Ostrik 2011, Corollary 5.21]) that the category $\operatorname{Rep}\left(S_{t}\right)$ is semisimple (and hence pre-Tannakian) for $t \notin \mathbb{Z}_{\geq 0}$. In particular, the category $\underline{\operatorname{Rep}}\left(S_{-1}\right)$ is pre-Tannakian, so its fundamental group $\pi$ is defined. For any $d \in \mathbb{Z}_{\geq 0}$ we can consider the commutative associative algebra with nondegenerate trace pairing $T_{d} \in \operatorname{Rep}\left(S_{-1}\right)$ which is a direct sum of [pt] and $d+1$ copies of the algebra $\mathbf{1}=[\varnothing]$. Clearly, $\operatorname{dim}\left(T_{d}\right)=d$, so we can use Proposition 1.1 to construct a symmetric tensor functor $\underline{\operatorname{Rep}}\left(S_{d}\right) \rightarrow \underline{\operatorname{Rep}}\left(S_{-1}\right)$. Using the general properties of the fundamental group we get a factorization of this functor as $\underline{\operatorname{Rep}}\left(S_{d}\right) \rightarrow \operatorname{Rep}\left(S_{\mathbf{I}}, \varepsilon\right) \rightarrow \underline{\operatorname{Rep}}\left(S_{-1}\right)$ (here $\mathbf{I}=\operatorname{Spec}\left(T_{d}\right)$ and $\varepsilon: \pi \rightarrow S_{\mathbf{I}}$ is the canonical homomorphism). It is clear that the category $\operatorname{Rep}\left(S_{\mathbf{I}}, \varepsilon\right)$ is pre-Tannakian; it is proved in [Deligne 2007, Proposition 8.19] that the functor $\underline{\operatorname{Rep}}\left(S_{d}\right) \rightarrow \operatorname{Rep}\left(S_{\mathbf{I}}, \varepsilon\right)$ is fully faithful. We set $\underline{\operatorname{Rep}}^{a b}\left(S_{d}\right):=\operatorname{Rep}\left(S_{\mathbf{I}}, \varepsilon\right)$; as explained above this is a pre-Tannakian category and we have a fully faithful braided tensor functor $\underline{\operatorname{Rep}}\left(S_{d}\right) \rightarrow \underline{\operatorname{Rep}}^{a b}\left(S_{d}\right)$.

Remark 2.5. The existence of the embedding $\underline{\operatorname{Rep}}\left(S_{t}\right) \subset \underline{\operatorname{Rep}}^{a b}\left(S_{t}\right)$ implies that $Y_{1} \otimes Y_{2} \neq 0$ for nonzero objects $Y_{1}, Y_{2} \in \underline{\operatorname{Rep}}\left(S_{t}\right)$ (this is true in any abelian rigid

\footnotetext{
${ }^{4}$ We refer the reader to [Deligne 2007, §1.7-1.8] for the discussion of this notion.
} 
tensor category with simple unit object). The same result can be proved directly as follows. Given finite sets $A$ and $B$, it follows from the definition of tensor products that the obvious map $\operatorname{End}([A]) \otimes \operatorname{End}([B]) \rightarrow \operatorname{End}([A] \otimes[B])=\operatorname{End}([A \sqcup B])$ is injective. Since any indecomposable object of $\underline{\operatorname{Rep}}\left(S_{t}\right)$ is the image of a primitive idempotent $e \in \operatorname{End}([A])$ for some finite set $A$ (see, e.g., [Comes and Ostrik 2011, Proposition 2.20]), it follows that the tensor product of two nonzero morphisms in $\underline{\operatorname{Rep}}\left(S_{t}\right)$ is nonzero. The statement for objects follows by considering their identity morphisms.

2B3. Indecomposable objects of the category $\operatorname{Rep}\left(S_{t}\right)$. The indecomposable objects of the category $\operatorname{Rep}\left(S_{t}\right)$ are classified up to isomorphism in [Comes and Ostrik 2011, Theorem 3.3]. The isomorphism classes are labeled by the Young diagrams of all sizes in the following way. Let $\lambda$ be a Young diagram of size $n=|\lambda|$ and let $y_{\lambda}$ be the corresponding primitive idempotent in $F S_{n}$, the group algebra of the symmetric group. ${ }^{5}$ The symmetric braiding gives rise to an action of $S_{n}$ on [pt $]^{\otimes n}$; let [pt $]^{\lambda}$ denote the image of $y_{\lambda} \in \operatorname{End}\left([\mathrm{pt}]^{\otimes n}\right)$. For any Young diagram $\lambda$ of size $|\lambda|$ there is a unique indecomposable object $L(\lambda) \in \underline{\operatorname{Rep}}\left(S_{t}\right)$ characterized by the following properties:

(a) $L(\lambda)$ is not a direct summand of [pt $]^{\otimes k}$ for $k<|\lambda|$.

(b) $L(\lambda)$ is a direct summand (with multiplicity 1 ) of $[\mathrm{pt}]^{\lambda}$.

It is proved in [Comes and Ostrik 2011, Theorem 3.3] that the indecomposable objects $L(\lambda)$ are well defined up to isomorphism, and any indecomposable object of $\underline{\operatorname{Rep}}\left(S_{t}\right)$ is isomorphic to precisely one $L(\lambda)$.

2B4. Blocks of the category $\underline{\operatorname{Rep}}\left(S_{t}\right)$. Let $\mathscr{A}$ be a Karoubian category such that any object decomposes into a finite direct sum of indecomposable objects. The set of isomorphism classes of indecomposable objects of $\mathscr{A}$ splits into blocks which are equivalence classes of the weakest equivalence relation for which two indecomposable objects are equivalent whenever there exists a nonzero morphism between them. We will also use the term block to refer to a full subcategory of $\mathscr{A}$ generated by the indecomposable objects in a single block.

The main result of [Comes and Ostrik 2011] is the description of blocks of the category $\underline{\operatorname{Rep}}\left(S_{t}\right)$. We describe the results of loc. cit. here. We will represent a Young diagram $\lambda$ as an infinite nonincreasing sequence $\left(\lambda_{1}, \lambda_{2}, \ldots\right)$ of nonnegative integers such that $\lambda_{k}=0$ for some $k>0$; see [Comes and Ostrik 2011, §1.1]. For a Young diagram $\lambda$ and $t \in F$ we define a sequence $\mu_{\lambda}(t)=\left(t-|\lambda|, \lambda_{1}-1, \lambda_{2}-2, \ldots\right)$.

Theorem 2.6 [Comes and Ostrik 2011, Theorem 5.3]. The objects $L(\lambda)$ and $L\left(\lambda^{\prime}\right)$ of $\underline{\operatorname{Rep}}\left(S_{t}\right)$ are in the same block if and only if $\mu_{\lambda}(t)$ is a permutation of $\mu_{\lambda^{\prime}}(t)$.

\footnotetext{
${ }^{5}$ Here $y_{\lambda}$ is a scalar multiple of the so-called Young symmetrizer (see, for instance, [Fulton and Harris 1991]).
} 
Let $\mathscr{B}$ be the set of blocks of the category $\underline{\operatorname{Rep}}\left(S_{t}\right)$; for any $\mathrm{b} \in \mathscr{B}$ let us denote by $\underline{\operatorname{Re}}_{\mathrm{b}}\left(S_{t}\right)$ the corresponding subcategory of $\underline{\operatorname{Rep}}\left(S_{t}\right)$; we have a decomposition $\underline{\operatorname{Rep}}\left(S_{t}\right)=\bigoplus_{\mathrm{b} \in \mathscr{B}} \underline{\operatorname{Rep}}_{\mathrm{b}}\left(S_{t}\right)$.

Proposition 2.7. Let $\mathrm{b} \in \mathscr{B}$. One of the following holds:

(i) $\mathrm{b}$ is semisimple (or trivial): the category $\underline{\operatorname{Rep}}_{\mathrm{b}}\left(S_{t}\right)$ is equivalent to the category $\operatorname{Vec}_{F}$ of finite-dimensional $F$-vector spaces as an additive category. We will denote by $L=L(\mathrm{~b})$ the unique indecomposable object of this block. Then $\operatorname{dim}(L)=0$, or, equivalently, $\operatorname{id}_{L}$ is negligible.

(ii) $\mathrm{b}$ is nonsemisimple (or infinite): in this case the additive category $\operatorname{Rep}_{\mathrm{b}}\left(S_{t}\right)$ is described in [Comes and Ostrik 2011, \$6] (in particular, it does not depend on a choice of nonsemisimple block $\mathrm{b}$ ). There is a natural labeling of indecomposable objects of the category $\operatorname{Re}_{\mathrm{b}}\left(S_{t}\right)$ by nonnegative integers; we will denote these objects by $L_{0}, L_{1}, \ldots$ Then $\operatorname{dim}\left(L_{i}\right)=0$ for $i>0$ and $\operatorname{dim}\left(L_{0}\right) \neq 0$, that is, $\mathrm{id}_{L_{i}}$ is negligible if and only if $i>0$.

Further, it is shown in [Comes and Ostrik 2011] that for any $t \in F$ there are infinitely many semisimple blocks and finitely many (precisely the number of Young diagrams of size $t$ ) nonsemisimple blocks. In particular, for $t \notin \mathbb{Z}_{\geq 0}$ all blocks are semisimple (hence the category $\underline{\operatorname{Rep}}\left(S_{t}\right)$ is semisimple).

2C. Temperley-Lieb category. The results on the category $\underline{\operatorname{Rep}}\left(S_{t}\right)$ in many respects are parallel to the results on the Temperley-Lieb category $T L(q)$. We recall the definition and some properties of this category here.

Definition 2.8 (see, for example, [Freedman 2003, §A1.2]). Let $q$ be a nonzero element of an algebraic closure of $F$ such that $q+q^{-1} \in F$. We define the $F$-linear tensor category $T L_{0}(q)$ as follows:

- Objects: finite subsets of $\mathbb{R}$ considered up to isotopy; we will denote the object corresponding to the set $A$ by $\langle A\rangle$.

- Morphisms: $\operatorname{Hom}(\langle A\rangle,\langle B\rangle)$ is the $F$-linear span of one-dimensional submanifolds of $\mathbb{R} \times[0,1]$ with boundary $A \sqcup B$ where $A \subset \mathbb{R} \times 0$ and $B \subset \mathbb{R} \times 1$ (such submanifolds are called embedded unoriented bordisms from $A$ to $B$ ) modulo the relation [bordism $\sqcup$ circle] $=\left(q+q^{-1}\right)$ [bordism]; composition is given by juxtaposition.

- Tensor product: disjoint union (write $\mathbb{R}=\mathbb{R}_{<0} \sqcup 0 \sqcup \mathbb{R}_{>0}$ and identify $\mathbb{R}_{<0}$ and $\mathbb{R}_{>0}$ with $\mathbb{R}$ ); the unit object is $\langle\varnothing\rangle$; tensor product of morphisms and associativity constraint are the obvious ones. 
Next we define the category $T L(q)$ as the Karoubian envelope of the category $T L_{0}(q)$. The category $T L(q)$ has a universal property (see, e.g., [Ostrik 2008, Theorem 2.4]) but we don't need it here. The indecomposable objects of the category $T L(q)$ are labeled by nonnegative integers: for any $i \in \mathbb{Z}_{\geq 0}$ there is a unique indecomposable object $V_{i}$ which is a direct summand (with multiplicity 1 ) of $\langle\mathrm{pt}\rangle^{\otimes i}$ but is not a direct summand of $\langle\mathrm{pt}\rangle^{\otimes k}$ whenever $k<i$.

The category $T L(q)$ is semisimple for generic values of $q$; more precisely the category $T L(q)$ is not semisimple precisely when there exists a positive integer $l$ such that $1+q^{2}+\cdots+q^{2 l}=0$ (we will denote the smallest such integer by $l_{q}$ ). Assume that the category $T L(q)$ is not semisimple. Then we have a full tensor functor $T L(q) \rightarrow \overline{\mathscr{C}}_{q}$ and a fully faithful tensor functor $T L(q) \rightarrow \mathscr{C}_{q}$, where $\overline{\mathscr{C}}_{q}$ is a semisimple tensor category (sometimes called the "Verlinde category") and $\mathscr{C}_{q}$ is the abelian tensor category of finite-dimensional representations of quantum SL(2); see, e.g., [Ostrik 2008, Theorem 2.4].

The blocks of the category $T L(q)$ are well known. Similarly to the case of the category $\underline{\operatorname{Rep}}\left(S_{d}\right)$ there are infinitely many semisimple blocks (which are equivalent to the category $\operatorname{Vec}_{F}$ as an additive category) and finitely many (precisely $l_{q}$ ) nonsemisimple blocks. The following observation is very important for this paper:

Proposition 2.9 [Comes and Ostrik 2011, Remark 6.5]. All nonsemisimple blocks of the category $T L(q)$ are equivalent as additive categories. Moreover, they are equivalent to the category $\operatorname{Re}_{\mathrm{b}}\left(S_{d}\right)$, where $\mathrm{b}$ is any nonsemisimple block of the category $\underline{\operatorname{Rep}}\left(S_{d}\right)$.

Remark 2.10. We can transport a labeling of indecomposable objects of $\underline{\operatorname{Rep}}_{\mathrm{b}}\left(S_{d}\right)$ (see Proposition 2.7(ii)) to a nonsemisimple block of the category $T L(q)$ via the equivalence of Proposition 2.9 (it is easy to see that the resulting labeling does not depend on a choice of the equivalence).

Recall that the category $T L(q)$ has a natural spherical structure and so the dimensions $\operatorname{dim}_{T L(q)}(Y)$ of objects $Y \in T L(q)$ are defined; see, e.g., [Freedman 2003, §A1.3]. The following result is well known; see, e.g., [Andersen 1992, (1.6) and Proposition 3.5]:

Lemma 2.11. Let $L$ be a unique indecomposable object in a semisimple block of $T L(q)$. Then $\operatorname{dim}_{T L(q)}(L)=0$. For a nonsemisimple block we have $\operatorname{dim}_{T L(q)}\left(L_{i}\right)=0$ for $i>0$ and $\operatorname{dim}_{T L(q)}\left(L_{0}\right) \neq 0$, where $L_{i}$ are indecomposable objects in this block labeled as in Remark 2.10.

\section{Tensor ideals and the object $\Delta \in \underline{\operatorname{Rep}}\left(S_{d}\right)$}

In this section we define objects $\Delta_{n} \in \underline{\operatorname{Rep}}\left(S_{t}\right)$ for $n \in \mathbb{Z}_{\geq 0}$ and $t \in F$. We then give $\Delta_{n}$ the structure of a commutative associative algebra in $\underline{\operatorname{Rep}}\left(S_{t}\right)$ and study many 
$\Delta_{n}$-modules. Finally, using our results on the objects $\Delta_{n}$, we classify tensor ideals in $\underline{\operatorname{Rep}}\left(S_{d}\right)$ when $d$ is a nonnegative integer. Before defining the objects $\Delta_{n}$ we prove the following easy observation which will be used throughout this section.

Proposition 3.1. Suppose $A_{0}, \ldots, A_{n}$ and $B_{0}, \ldots, B_{m}$ are finite sets with $A_{0}=B_{0}$ and $A_{n}=B_{m}$. Suppose further that $f_{i}$ (resp. $g_{i}$ ) is an F-linear combination of partitions of $A_{i-1} \sqcup A_{i}$ (resp. $B_{i-1} \sqcup B_{i}$ ) whose coefficients do not depend on $t$ for all $1 \leq i \leq n$ (resp. $1 \leq i \leq m$ ). If $f_{n} \cdots f_{1}=g_{m} \cdots g_{1}$ in $\underline{\operatorname{Rep}}_{0}\left(S_{t}\right)$ for infinitely many values of $t \in F$, then $f_{n} \cdots f_{1}=g_{m} \cdots g_{1}$ in $\underline{\operatorname{Rep}}_{0}\left(S_{t}\right)$ for all $t \in F$.

Proof. For each $t \in F$ and partition $\pi$ of $A_{0} \sqcup A_{n}=B_{0} \sqcup B_{m}$, let $a_{\pi}(t) \in F$ (resp. $b_{\pi}(t) \in F$ ) be such that $f_{n} \cdots f_{1}=\sum_{\pi} a_{\pi}(t) \pi$ (resp. $g_{m} \cdots g_{1}=\sum_{\pi} b_{\pi}(t) \pi$ ) in $\underline{\operatorname{Rep}}_{0}\left(S_{t}\right)$ where the sum is taken over all partitions $\pi$ of $A_{0} \sqcup A_{n}=B_{0} \sqcup B_{m}$. Then $f_{n} \cdots f_{1}=g_{m} \cdots g_{1}$ in $\underline{\operatorname{Rep}}_{0}\left(S_{t}\right)$ if and only if $a_{\pi}(t)=b_{\pi}(t)$ for all $\pi$. By the definition of composition in $\operatorname{Rep}_{0}\left(S_{t}\right)$, both $a_{\pi}(t)$ and $b_{\pi}(t)$ are polynomials in $t$ for each $\pi$. The result follows since a polynomial in $t$ is determined by finitely many values of $t$.

3A. The objects $\Delta_{n} \in \operatorname{Rep}\left(S_{t}\right)$. Suppose $n$ is a nonnegative integer and let $A_{n}=$ $\{i \mid 1 \leq i \leq n\}$. Consider the endomorphism $x_{n}=x_{\mathrm{id}_{n}}:\left[A_{n}\right] \rightarrow\left[A_{n}\right]$ in $\underline{\operatorname{Rep}}_{0}\left(S_{t}\right)$ (see [Comes and Ostrik 2011, Equation (2.1)]).

Proposition 3.2. $x_{n}$ is an idempotent which is equal to its dual for all $n \geq 0$.

Proof. The fact that $x_{n}^{*}=x_{n}$ follows from the definition of $x_{n}$. By Proposition 3.1, it suffices to show $x_{n}$ is an idempotent in $\underline{\operatorname{Rep}}_{0}\left(S_{t}\right)$ for infinitely many values of $t$. It follows from [Comes and Ostrik 2011, Theorem 2.6 and Equation (2.2)] that $x_{n}$ is an idempotent in $\underline{\operatorname{Rep}}_{0}\left(S_{t}\right)$ whenever $t$ is an integer greater than $2 n$.

Since $\operatorname{Rep}\left(S_{t}\right)$ is a Karoubian category (i.e., $\operatorname{Rep}\left(S_{t}\right)$ contains images of idempotents) the following definition is valid.

Definition 3.3. Let $\Delta_{n} \in \underline{\operatorname{Rep}}\left(S_{t}\right)$ denote the image of the idempotent $x_{n}{ }^{6}$

Note that the commutative associative algebra structure on [pt] extends in an obvious way to a commutative associative algebra structure on $\left[A_{n}\right] \cong[\mathrm{pt}]^{\otimes n}$. Let $\mu_{n}:\left[A_{n}\right] \otimes\left[A_{n}\right] \rightarrow\left[A_{n}\right]$ be the multiplication map and $1_{n}: \mathbf{1} \rightarrow\left[A_{n}\right]$ the unit map.

Proposition 3.4. The multiplication map $x_{n} \mu_{n}\left(x_{n} \otimes x_{n}\right): \Delta_{n} \otimes \Delta_{n} \rightarrow \Delta_{n}$ gives $\Delta_{n}$ the structure of a commutative associative algebra in $\operatorname{Rep}\left(S_{t}\right)$ with unit given by $x_{n} 1_{n}: \mathbf{1} \rightarrow \Delta_{n}$.

${ }^{6}$ In the notation of [Comes and Ostrik 2011], $\Delta_{n}=\left([n], x_{n}\right)$. 
Proof. We are required to show the following equalities hold in $\underline{\operatorname{Rep}}_{0}\left(S_{t}\right)$ :

$$
\begin{aligned}
x_{n} \mu_{n}\left(x_{n} \mu_{n}\left(x_{n} \otimes x_{n}\right) \otimes x_{n}\right) & =x_{n} \mu_{n}\left(x_{n} \otimes\left(x_{n} \mu_{n}\left(x_{n} \otimes x_{n}\right)\right),\right. \\
x_{n} \mu_{n}\left(x_{n} 1_{n} \otimes x_{n}\right) & =x_{n}=x_{n} \mu_{n}\left(x_{n} \otimes x_{n} 1_{n}\right), \\
x_{n} \mu_{n}\left(x_{n} \otimes x_{n}\right) \beta_{n, n}\left(x_{n} \otimes x_{n}\right) & =x_{n} \mu_{n}\left(x_{n} \otimes x_{n}\right),
\end{aligned}
$$

where $\beta_{n, n}: A_{n} \otimes A_{n} \rightarrow A_{n} \otimes A_{n}$ is the braiding morphism (see for example [Comes and Ostrik 2011, §2.2]). By Proposition 3.1, it suffices to show (3-1), (3-2) and (3-3) hold for infinitely many values of $t .^{7}$ Using Theorem 2.6 and Equation (2.2) of the same reference it is easy to show (3-1), (3-2) and (3-3) hold whenever $t$ is a sufficiently large integer.

By Proposition 3.4 we can consider the category $\Delta_{n}$-mod of all left $\Delta_{n}$-modules.

3B. Some $\Delta_{n}$-modules. Suppose $j$ is a nonnegative integer with $1 \leq j \leq n$. Given a finite set $X$, let $\Theta_{X}^{j}: \operatorname{Hom}_{\underline{R e p}\left(S_{t}\right)}\left(A_{n}, X\right) \rightarrow \operatorname{Hom}_{\underline{\operatorname{Rep}\left(S_{t}\right)}}\left(A_{n+1}, X\right)$ and $\Theta_{j}^{X}: \operatorname{Hom}_{\underline{\operatorname{Rep}}\left(S_{t}\right)}\left(X, A_{n}\right) \rightarrow \operatorname{Hom}_{\underline{\operatorname{Rep}}\left(S_{t}\right)}\left(X, A_{n+1}\right)$ be the $F$-linear maps defined on partitions as follows: if $\pi$ is a partition of $X \sqcup A_{n}$, then $\Theta_{X}^{j}(\pi)=\Theta_{j}^{X}(\pi)$ is the unique partition of $X \sqcup A_{n+1}$ which restricts to $\pi$ and has $j$ and $n+1$ in the same part. Now let $\Theta_{j}: \operatorname{End}_{\left.\underline{\operatorname{Rep}\left(S_{t}\right.}\right)}\left(A_{n}\right) \rightarrow \operatorname{End}_{\underline{\operatorname{Rep}\left(S_{t}\right)}}\left(A_{n+1}\right)$ be the $F$-linear map $\Theta_{j}=\Theta_{A_{n}}^{j} \circ \Theta_{j}^{A_{n}}$. It is easy to check that $\Theta_{j}$ is an injective (nonunital) $F$-algebra homomorphism for each $1 \leq j \leq n$. In particular, by Proposition 3.2, $x_{n, j}:=\Theta_{j}\left(x_{n}\right)$ is an idempotent for each $j$.

Definition 3.5. Let $\Delta_{n}(j) \in \underline{\operatorname{Rep}}\left(S_{t}\right)$ denote the image of $x_{n, j}$.

Next we give $\Delta_{n}(j)$ the structure of a $\Delta_{n}$-module. Let

$$
\begin{aligned}
& \alpha=x_{n, j} \Theta_{j}^{A_{n}}\left(x_{n}\right) x_{n}: \Delta_{n} \rightarrow \Delta_{n}(j), \\
& \beta=x_{n, j} \Theta_{j}\left(\mu_{n}\right)\left(x_{n, j} \otimes x_{n, j}\right): \Delta_{n}(j) \otimes \Delta_{n}(j) \rightarrow \Delta_{n}(j) .
\end{aligned}
$$

Finally, let $\phi=\beta\left(\alpha \otimes x_{n, j}\right): \Delta_{n} \otimes \Delta_{n}(j) \rightarrow \Delta_{n}(j)$.

Proposition 3.6. (1) The map $\phi$ gives $\Delta_{n}(j)$ the structure of $a \Delta_{n}$-module.

(2) The map $x_{n, j} \Theta_{j}^{A_{n}}\left(\mathrm{id}_{A_{n}}\right) x_{n}: \Delta_{n} \rightarrow \Delta_{n}(j)$ is an isomorphism of $\Delta_{n}$-modules with inverse $x_{n} \Theta_{A_{n}}^{j}\left(\mathrm{id}_{A_{n}}\right) x_{n, j}$.

Proof. For part (1) we are required to show the following equation holds in $\underline{\operatorname{Rep}}_{0}\left(S_{t}\right)$ :

$$
\begin{aligned}
& x_{n, j} \Theta_{j}\left(\mu_{n}\right)\left(\left(x_{n, j} \Theta_{j}^{A_{n}}\left(x_{n}\right) x_{n} \mu_{n}\left(x_{n} \otimes x_{n}\right)\right) \otimes x_{n, j}\right) \\
& =x_{n, j} \Theta_{j}\left(\mu_{n}\right)\left(x_{n, j} \Theta_{j}^{A_{n}}\left(x_{n}\right) x_{n} \otimes\left(x_{n, j} \Theta_{j}\left(\mu_{n}\right)\left(\left(x_{n, j} \Theta_{j}^{A_{n}}\left(x_{n}\right) x_{n}\right) \otimes x_{n, j}\right)\right) .\right.
\end{aligned}
$$

\footnotetext{
${ }^{7}$ In fact, (3-1), (3-2) and (3-3) do not depend on $t$, so we only need to verify they hold for some $t$.
} 
For part (2) we are required to show the following equations hold in $\underline{\operatorname{Rep}}_{0}\left(S_{t}\right)$ :

$$
\begin{gathered}
x_{n, j} \Theta_{j}^{A_{n}}\left(\mathrm{id}_{A_{n}}\right) x_{n} \Theta_{A_{n}}^{j}\left(\mathrm{id}_{A_{n}}\right) x_{n, j}=x_{n, j}, \\
x_{n} \Theta_{A_{n}}^{j}\left(\mathrm{id}_{A_{n}}\right) x_{n, j} \Theta_{j}^{A_{n}}\left(\mathrm{id}_{A_{n}}\right) x_{n}=x_{n} .
\end{gathered}
$$

Now use Proposition 3.1 and [Comes and Ostrik 2011, Theorem 2.6 and Equation (2.2)].

Next, we give the object $\Delta_{n+1}$ the structure of a $\Delta_{n}$-module. To do so, set $\psi=x_{n+1}\left(\mu_{n} \otimes \operatorname{id}_{[\mathrm{pt}]}\right)\left(x_{n} \otimes x_{n+1}\right): \Delta_{n} \otimes \Delta_{n+1} \rightarrow \Delta_{n+1}$.

Proposition 3.7. The map $\psi$ gives $\Delta_{n+1}$ the structure of a $\Delta_{n}$-module.

Proof. We are required to show the following equation holds in $\operatorname{Rep}_{0}\left(S_{t}\right)$ :

$$
\begin{aligned}
x_{n+1}\left(\mu_{n} \otimes \mathrm{id}_{[\mathrm{pt}]}\right) & \left(\left(x_{n} \mu_{n}\left(x_{n} \otimes x_{n}\right)\right) \otimes x_{n+1}\right) \\
= & x_{n+1}\left(\mu_{n} \otimes \mathrm{id}_{[\mathrm{pt}]}\right)\left(x_{n} \otimes\left(x_{n+1}\left(\mu_{n} \otimes \mathrm{id}_{[\mathrm{pt}]}\right)\left(x_{n} \otimes x_{n+1}\right)\right)\right) .
\end{aligned}
$$

Now use Proposition 3.1 and [Comes and Ostrik 2011, Theorem 2.6 and Equation (2.6)].

The following lemma will be important for us later.

Lemma 3.8. $\Delta_{n} \otimes[\mathrm{pt}] \cong \Delta_{n+1} \oplus \Delta_{n}(1) \oplus \cdots \oplus \Delta_{n}(n)$ in the category $\Delta_{n}$-mod.

Proof. First, using Proposition 3.1 and [Comes and Ostrik 2011, Theorem 2.6 and Equation (2.6)] it is easy to show that the following identities hold in $\underline{\operatorname{Re}}_{0}\left(S_{t}\right)$ :

$$
\begin{aligned}
x_{n} \otimes \mathrm{id}_{[\mathrm{pt}]} & =x_{n+1}+\sum_{1 \leq j \leq n} x_{n, j}, & & \\
x_{n, j} x_{n+1} & =0=x_{n+1} x_{n, j} & & (1 \leq j \leq n), \\
x_{n, j} x_{n, k} & =\delta_{j, k} x_{n, j} & & (1 \leq j, k \leq n) .
\end{aligned}
$$

Next, define $\Psi: \Delta_{n} \otimes[\mathrm{pt}] \rightarrow \Delta_{n+1} \oplus \Delta_{n}(1) \oplus \cdots \oplus \Delta_{n}(n)$ by

$$
\Psi=\left[\begin{array}{c}
x_{n+1}\left(x_{n} \otimes \mathrm{id}_{[\mathrm{pt}]}\right) \\
x_{n, 1}\left(x_{n} \otimes \mathrm{id}_{[\mathrm{pt}]}\right) \\
\vdots \\
x_{n, n}\left(x_{n} \otimes \mathrm{id}_{[\mathrm{pt}]}\right)
\end{array}\right] .
$$

Using (3-8) it is easy to check that $\Psi$ is an isomorphism in $\underline{\operatorname{Rep}}\left(S_{t}\right)$ with inverse

$$
\Psi^{-1}=\left[\left(x_{n} \otimes \mathrm{id}_{[\mathrm{pt}]}\right) x_{n+1} \quad\left(x_{n} \otimes \mathrm{id}_{[\mathrm{pt}]}\right) x_{n, 1} \cdots \quad\left(x_{n} \otimes \mathrm{id}_{[\mathrm{pt}]}\right) x_{n, n}\right] .
$$

It remains to show that $\Psi$ and $\Psi^{-1}$ are morphisms in the category $\Delta_{n}$-mod. Showing that $\Psi$ is a morphism in $\Delta_{n}$-mod amounts to showing the following equations hold 
in $\underline{\operatorname{Rep}}_{0}\left(S_{t}\right)$ :

$$
\begin{aligned}
& x_{n+1}\left(\mu_{n} \otimes \mathrm{id}_{[\mathrm{pt}]}\right)\left(x_{n} \otimes\left(x_{n+1}\left(x_{n} \otimes \mathrm{id}_{[\mathrm{pt}]}\right)\right)\right) \\
& =x_{n+1}\left(\left(x_{n} \mu_{n}\left(x_{n} \otimes x_{n}\right)\right) \otimes \mathrm{id}_{[\mathrm{pt}]}\right), \\
& x_{n, j} \Theta_{j}\left(\mu_{n}\right)\left(\left(x_{n, j} \Theta_{j}^{A_{n}}\left(x_{n}\right) x_{n}\right) \otimes\left(x_{n, j}\left(x_{n} \otimes \mathrm{id}_{[\mathrm{pt}]}\right)\right)\right) \\
& =x_{n, j}\left(\left(x_{n} \mu_{n}\left(x_{n} \otimes x_{n}\right)\right) \otimes \mathrm{id}_{[\mathrm{pt}]}\right) \quad(1 \leq j \leq n) .
\end{aligned}
$$

To show the equations in (3-11) hold, use Proposition 3.1 and [Comes and Ostrik 2011, Theorem 2.6 and Equation (2.6)]. The proof for $\Psi^{-1}$ is similar.

3C. The category $\operatorname{Rep}^{\Delta_{n}}\left(S_{t}\right)$. Let $\Delta_{n}-\bmod _{0}$ denote the full subcategory of $\Delta_{n}$ mod such that a $\Delta_{n}$-module $M$ is in $\Delta_{n}-\bmod _{0}$ if and only if $M \cong \Delta_{n} \otimes Y$ in $\Delta_{n}$-mod for some $Y \in \underline{\operatorname{Rep}}\left(S_{t}\right)$. Let $\underline{\operatorname{Rep}}^{\Delta_{n}}\left(S_{t}\right)$ denote the Karoubian envelope of $\Delta_{n}-\bmod _{0}$. The advantage of working in $\operatorname{Rep}^{\Delta_{n}}\left(S_{t}\right)$ rather than in the category $\Delta_{n}$-mod is that we can give $\underline{\operatorname{Rep}}^{\Delta_{n}}\left(S_{t}\right)$ the structure of a tensor category with relative ease. Indeed, given $M, M^{\prime} \in \Delta_{n}-\bmod _{0}$ we know $M \cong \Delta_{n} \otimes Y$ and $M^{\prime} \cong \Delta_{n} \otimes Y^{\prime}$ as $\Delta_{n}$-modules for some $Y, Y^{\prime} \in \underline{\operatorname{Rep}}\left(S_{t}\right)$. Set $M \otimes_{\Delta_{n}} M^{\prime}:=\Delta_{n} \otimes Y \otimes Y^{\prime}$. Given $N, N^{\prime} \in \Delta_{n}-\bmod _{0}$ with $N \cong \Delta_{n} \otimes Z$ and $N^{\prime} \cong \Delta_{n} \otimes Z^{\prime}$ and morphisms $f \in \operatorname{Hom}_{\Delta_{n}-\bmod _{0}}(M, N)$ and $g \in \operatorname{Hom}_{\Delta_{n}-\bmod _{0}}\left(M^{\prime}, N^{\prime}\right)$, write

$$
\begin{aligned}
& \tilde{f}: \Delta_{n} \otimes Y \stackrel{\cong}{\rightarrow} M \stackrel{f}{\rightarrow} N \stackrel{\cong}{\rightarrow} \Delta_{n} \otimes Z, \\
& \tilde{g}: \Delta_{n} \otimes Y^{\prime} \stackrel{\cong}{\rightarrow} M^{\prime} \stackrel{g}{\rightarrow} N^{\prime} \stackrel{\cong}{\rightarrow} \Delta_{n} \otimes Z^{\prime} .
\end{aligned}
$$

Define $f \otimes_{\Delta_{n}} g: M \otimes_{\Delta_{n}} M^{\prime} \rightarrow N \otimes_{\Delta_{n}} N^{\prime}$ to be the composition

$$
\begin{aligned}
M \otimes_{\Delta_{n}} M^{\prime}=\Delta_{n} \otimes Y \otimes Y^{\prime} & \stackrel{\tilde{f} \otimes \mathrm{id}_{Y^{\prime}}}{\longrightarrow} \Delta_{n} \otimes Z \otimes Y^{\prime} \stackrel{\sim}{\rightarrow} \Delta_{n} \otimes Y^{\prime} \otimes Z \\
& \stackrel{\tilde{g} \otimes \mathrm{id}_{Z}}{\longrightarrow} \Delta_{n} \otimes Z^{\prime} \otimes Z \stackrel{\sim}{\rightarrow} \Delta_{n} \otimes Z \otimes Z^{\prime}=N \otimes_{\Delta_{n}} N^{\prime} .
\end{aligned}
$$

It is easy to check that $\otimes_{\Delta_{n}}: \Delta_{n}-\bmod _{0} \times \Delta_{n}-\bmod _{0} \rightarrow \Delta_{n}-\bmod _{0}$ is a bifunctor which (with the obvious choice of constraints) makes $\Delta_{n}-\bmod _{0}$ into a rigid symmetric tensor category. The tensor structure on $\Delta_{n}-\bmod _{0}$ extends in an obvious way to make $\operatorname{Rep}^{\Delta_{n}}\left(S_{t}\right)$ a rigid symmetric tensor category too.

Notice that $\Delta_{n+1}$ is an object in $\operatorname{Rep}^{\Delta_{n}}\left(S_{t}\right)$. Indeed, by Lemma 3.8, the $\Delta_{n}$ module $\Delta_{n+1}$ is the image of an idempotent of the form $\Delta_{n} \otimes[\mathrm{pt}] \rightarrow \Delta_{n} \otimes[\mathrm{pt}]$. This idempotent is an element of $\operatorname{End}_{\Delta_{n}-\bmod _{0}}\left(\Delta_{n} \otimes[\mathrm{pt}]\right)$; hence its image is an object in the Karoubian category $\operatorname{Rep}^{\Delta_{n}}\left(S_{t}\right)$. The next two propositions concern the structure of $\Delta_{n+1} \in \underline{\operatorname{Rep}}^{\Delta_{n}}\left(S_{t}\right)$. We start by computing its dimension:

$$
\text { Proposition 3.9. } \quad \operatorname{dim}_{\underline{\operatorname{Rep}}^{\Delta_{n}\left(S_{t}\right)}}\left(\Delta_{n+1}\right)=t-n \text {. }
$$

Proof. First, by Lemma 3.8 and Proposition 3.6(2),

$$
\operatorname{dim}_{\underline{\operatorname{Rep}}^{\Delta_{n}}\left(S_{t}\right)}\left(\Delta_{n+1}\right)=\operatorname{dim}_{\underline{\operatorname{Rep}}^{\Delta_{n}}\left(S_{t}\right)}\left(\Delta_{n} \otimes[\mathrm{pt}]\right)-n \operatorname{dim}_{\underline{\operatorname{Rep}}^{\Delta_{n}}\left(S_{t}\right)}\left(\Delta_{n}\right) .
$$


Now, consider the tensor functor $\Delta_{n} \otimes-: \underline{\operatorname{Rep}}\left(S_{t}\right) \rightarrow \underline{\operatorname{Rep}}^{\Delta_{n}}\left(S_{t}\right)$. Since ten-

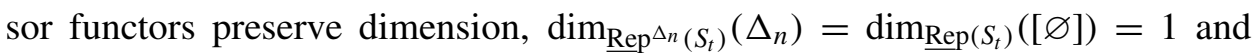

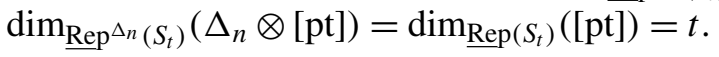

Our next aim is to show $\Delta_{n+1} \in \operatorname{Rep}^{\Delta_{n}}\left(S_{t}\right)$ satisfies (a) and (b) from Section 1B. To do so, let inc : $\Delta_{n+1} \rightarrow \Delta_{n} \otimes[\mathrm{pt}]$ and proj : $\Delta_{n} \otimes[\mathrm{pt}] \rightarrow \Delta_{n+1}$ denote the morphisms in $\operatorname{Rep}^{\Delta_{n}}\left(S_{t}\right)$ determined by Lemma 3.8. Moreover, let

$$
m:\left(\Delta_{n} \otimes[\mathrm{pt}]\right) \otimes_{\Delta_{n}}\left(\Delta_{n} \otimes[\mathrm{pt}]\right) \rightarrow \Delta_{n} \otimes[\mathrm{pt}]
$$

denote the morphism $\Delta_{n} \otimes[\mathrm{pt}] \otimes[\mathrm{pt}] \stackrel{\mathrm{id}_{\Delta_{n}} \otimes \mu_{1}}{\longrightarrow} \Delta_{n} \otimes[\mathrm{pt}]$. Now consider the morphisms

$$
\Delta_{n+1} \otimes_{\Delta_{n}} \Delta_{n+1} \stackrel{\text { inc } \otimes_{\Delta_{n}} \text { inc }}{\longrightarrow}\left(\Delta_{n} \otimes[\mathrm{pt}]\right) \otimes_{\Delta_{n}}\left(\Delta_{n} \otimes[\mathrm{pt}]\right) \stackrel{m}{\longrightarrow} \Delta_{n} \otimes[\mathrm{pt}] \stackrel{\text { proj }}{\longrightarrow} \Delta_{n+1}
$$

and

$$
\Delta_{n} \stackrel{\mathrm{id}_{\Delta_{n}} \otimes 1_{1}}{\longrightarrow} \Delta_{n} \otimes[\mathrm{pt}] \stackrel{\text { proj }}{\longrightarrow} \Delta_{n+1} .
$$

Proposition 3.10. With the multiplication and unit maps given by (3-12) and (3-13) respectively, $\Delta_{n+1} \in \underline{\operatorname{Rep}}^{\Delta_{n}}\left(S_{t}\right)$ satisfies (a) and (b) from Section $1 B$.

Proof. Write $\mu_{\Delta_{n+1}}$ and $1_{\Delta_{n+1}}$ for the morphisms given by (3-12) and (3-13) respectively. First, it is easy to see that $m$ (resp. $\mathrm{id}_{\Delta_{n}} \otimes 1_{1}$ ) is a morphism of $\Delta_{n}$-modules. Hence, $\mu_{\Delta_{n+1}}$ (resp. $1_{\Delta_{n+1}}$ ) is a morphism of $\Delta_{n}$-modules too. Now, to show $\Delta_{n+1}$ satisfies (a) from Section 1B we must show the following equations hold in $\operatorname{Rep}^{\Delta_{n}}\left(S_{t}\right)$ :

$$
\begin{aligned}
\mu_{\Delta_{n+1}}\left(\mu_{\Delta_{n+1}} \otimes_{\Delta_{n}} \mathrm{id}_{\Delta_{n+1}}\right) & =\mu_{\Delta_{n+1}}\left(\mathrm{id}_{\Delta_{n+1}} \otimes_{\Delta_{n}} \mu_{\Delta_{n+1}}\right), \\
\mu_{\Delta_{n+1}}\left(1_{\Delta_{n+1}} \otimes_{\Delta_{n}} \operatorname{id}_{\Delta_{n+1}}\right) & =\operatorname{id}_{\Delta_{n+1}}=\mu_{\Delta_{n+1}}\left(\operatorname{id}_{\Delta_{n+1}} \otimes_{\Delta_{n}} 1_{\Delta_{n+1}}\right), \\
\mu_{\Delta_{n+1}} \beta_{\Delta_{n+1}, \Delta_{n+1}} & =\mu_{\Delta_{n+1}},
\end{aligned}
$$

where $\beta_{\Delta_{n+1}, \Delta_{n+1}}: \Delta_{n+1} \otimes_{\Delta_{n}} \Delta_{n+1} \rightarrow \Delta_{n+1} \otimes_{\Delta_{n}} \Delta_{n+1}$ denotes the braiding morphism. To do so, first notice that by (3-8) the morphisms proj, inc, and $\mathrm{id}_{\Delta_{n+1}}$ are all given by $x_{n+1}$. Let $\tau$ (resp. $v$ ) denote the identity morphism on $\Delta_{n+1} \otimes_{\Delta_{n}} \Delta_{n+1}$ (resp. $\Delta_{n+1} \otimes_{\Delta_{n}} \Delta_{n+1} \otimes_{\Delta_{n}} \Delta_{n+1}$ ). Then, by the definition of $\otimes_{\Delta_{n}}$, we have the following realizations of $\tau$ and $v$ as morphisms in $\underline{\operatorname{Rep}}_{0}\left(S_{t}\right)$ :

$$
\begin{aligned}
& \tau=\left(x_{n} \otimes \beta_{1,1}\right)\left(x_{n+1} \otimes \mathrm{id}_{[\mathrm{pt}]}\right)\left(x_{n} \otimes \beta_{1,1}\right)\left(x_{n+1} \otimes \mathrm{id}_{[\mathrm{pt}]}\right), \\
& v=\left(x_{n} \otimes \beta_{1,2}\right)\left(x_{n+1} \otimes \mathrm{id}_{[\mathrm{pt}] \otimes[\mathrm{pt}]}\right)\left(x_{n} \otimes \beta_{2,1}\right)\left(\tau \otimes \mathrm{id}_{[\mathrm{pt}]}\right),
\end{aligned}
$$

where $\beta_{n, m}: A_{n} \otimes A_{m} \rightarrow A_{m} \otimes A_{n}$ denotes the braiding morphism in $\underline{\operatorname{Rep}}_{0}\left(S_{t}\right)$ for each $n, m \geq 0$. Moreover,

$$
\begin{gathered}
1_{\Delta_{n+1}}=x_{n+1}\left(x_{n} \otimes 1_{1}\right), \quad \mu_{\Delta_{n+1}}=x_{n+1}\left(x_{n} \otimes \mu_{1}\right) \tau, \\
\beta_{\Delta_{n+1}, \Delta_{n+1}}=\tau\left(x_{n} \otimes \beta_{1,1}\right) \tau .
\end{gathered}
$$


Thus, showing the equations in (3-14) hold in $\operatorname{Rep}^{\Delta_{n}}\left(S_{t}\right)$ amounts to showing the following equations hold in $\underline{\operatorname{Re}}_{0}\left(S_{t}\right)$ :

$$
\begin{aligned}
& x_{n+1}\left(x_{n} \otimes \mu_{1}\right) \tau\left(x_{n} \otimes \beta_{1,1}\right)\left(x_{n+1} \otimes \mathrm{id}_{[\mathrm{pt}]}\right)\left(x_{n} \otimes \beta_{1,1}\right)\left(\left(x_{n+1}\left(x_{n} \otimes \mu_{1}\right) \tau\right) \otimes \mathrm{id}_{[\mathrm{pt}]}\right) v \\
& =x_{n+1}\left(x_{n} \otimes \mu_{1}\right) \tau\left(x_{n} \otimes \beta_{1,1}\right)\left(\left(x_{n+1}\left(x_{n} \otimes \mu_{1}\right) \tau\right) \otimes \mathrm{id}_{[\mathrm{pt}]}\right)\left(x_{n} \otimes \beta_{1,2}\right)\left(x_{n+1} \otimes \mathrm{id}_{[\mathrm{pt}] \otimes[\mathrm{pt}]}\right) v \text {, } \\
& x_{n+1}\left(x_{n} \otimes \mu_{1}\right) \tau\left(x_{n} \otimes \beta_{1,1}\right)\left(x_{n+1} \otimes \mathrm{id}_{[\mathrm{pt}]}\right)\left(x_{n} \otimes \beta_{1,1}\right)\left(x_{n+1}\left(x_{n} \otimes 1_{1}\right) \otimes \mathrm{id}_{[\mathrm{pt}]}\right) \\
& =x_{n+1}=x_{n+1}\left(x_{n} \otimes \mu_{1}\right) \tau\left(x_{n+1} \otimes \beta_{1,1}\right)\left(\left(x_{n+1}\left(x_{n} \otimes 1_{1}\right)\right) \otimes \mathrm{id}_{[\mathrm{pt}]}\right) x_{n+1} \text {, } \\
& x_{n+1}\left(x_{n} \otimes \mu_{1}\right) \tau\left(x_{n} \otimes \beta_{1,1}\right) \tau=x_{n+1}\left(x_{n} \otimes \mu_{1}\right) \tau \text {. }
\end{aligned}
$$

All equations above are straightforward to check using Proposition 3.1 and [Comes and Ostrik 2011, Theorem 2.6 and Equation (2.6)]. Thus $\Delta_{n+1}$ satisfies part (a) from Section 1B.

To show $\Delta_{n+1}$ satisfies Section $1 \mathrm{~B}(\mathrm{~b})$, first notice that $\Delta_{n+1} \in \operatorname{Rep}^{\Delta_{n}}\left(S_{t}\right)$ is self-dual (because the morphism $x_{n+1}$ is self-dual). Hence, we are required to show that the following morphism is invertible in $\operatorname{Rep}^{\Delta_{n}}\left(S_{t}\right)$ :

$$
\left(\left(\operatorname{Tr} \mu_{\Delta_{n+1}}\right) \otimes_{\Delta_{n}} \mathrm{id}_{\Delta_{n+1}}\right)\left(\mathrm{id}_{\Delta_{n+1}} \otimes_{\Delta_{n}} \operatorname{coev}_{\Delta_{n+1}}\right): \Delta_{n+1} \rightarrow \Delta_{n+1},
$$

where the morphism $\operatorname{Tr}: \Delta_{n+1} \rightarrow \Delta_{n}$ is defined in Section $1 \mathrm{~B}(\mathrm{~b})$. In fact, we claim the morphism in (3-17) is equal to the identity morphism $\operatorname{id}_{\Delta_{n+1}}$. To prove this claim, first notice that

$$
\operatorname{Tr}=\operatorname{ev}_{\Delta_{n+1}} \beta_{\Delta_{n+1}, \Delta_{n+1}}\left(\mu_{\Delta_{n+1}} \otimes_{\Delta_{n}} \operatorname{id}_{\Delta_{n+1}}\right)\left(\operatorname{id}_{\Delta_{n+1}} \otimes_{\Delta_{n}} \operatorname{coev}_{\Delta_{n+1}}\right) .
$$

Also, $\mathrm{ev}_{\Delta_{n+1}}=x_{n}\left(x_{n} \otimes \mathrm{ev}_{[\mathrm{pt}]}\right) \tau$ and $\operatorname{coev}_{\Delta_{n+1}}=\tau\left(x_{n} \otimes \operatorname{coev}_{[\mathrm{pt}]}\right) x_{n}$. Hence, using (3-15), (3-16), and the definition of $\otimes_{\Delta_{n}}$, we can realize the morphism in (3-17) as a morphism in $\underline{\operatorname{Rep}}_{0}\left(S_{t}\right)$. Now use Proposition 3.1 and [Comes and Ostrik 2011, Theorem 2.6 and Equation (2.6)] to show that this morphism is equal to $x_{n+1}$.

3D. Deligne's lemma. Fix an integer $d \geq 0$. Set $\Delta=\Delta_{d+1} \in \underline{\operatorname{Rep}}\left(S_{d}\right)$ and $\Delta^{+}=\Delta_{d+2} \in \underline{\operatorname{Rep}}^{\Delta}\left(S_{d}\right)$. By Proposition 3.9, $\operatorname{dim}_{\underline{\operatorname{Rep}}^{\Delta}\left(S_{d}\right)}\left(\Delta^{+}\right)=-1$. Hence, by

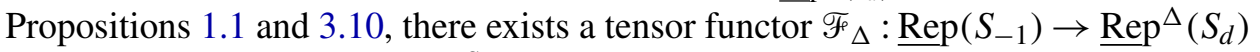
with $\mathscr{F}_{\Delta}([\mathrm{pt}])=\Delta^{+}$. Let $\underline{\operatorname{Res}}_{S_{-1}}^{S_{d}}$ denote the tensor functor $\underline{\operatorname{Rep}}\left(S_{d}\right) \rightarrow \underline{\operatorname{Rep}}\left(S_{-1}\right)$ described in Definition 2.4, i.e., the functor prescribed by Proposition 1.1 with $\underline{\operatorname{Res}}_{S_{-1}}^{S_{d}}([\mathrm{pt}])=[\mathrm{pt}] \oplus[\varnothing]^{\oplus d+1}$. Then we have the following:

Lemma 3.11. The functor $\Delta \otimes-: \underline{\operatorname{Rep}}\left(S_{d}\right) \rightarrow \underline{\operatorname{Rep}}^{\Delta}\left(S_{d}\right)$ is isomorphic to the composition $\mathscr{F}_{\Delta} \circ \underline{\operatorname{Res}_{S}} S_{-1}$.

Proof. Both $\Delta \otimes-$ and $\mathscr{F}_{\Delta} \circ \underline{\operatorname{Res}}_{S_{-1}}^{S_{d}}$ are tensor functors which map [pt] $\in \underline{\operatorname{Rep}}\left(S_{d}\right)$ to an object isomorphic to $\Delta^{+} \oplus \Delta^{\oplus d+1} \in \underline{\operatorname{Rep}}^{\Delta}\left(S_{d}\right)$ (see Propositions 3.6(2) and 3.8). Hence, by Proposition 1.1, they are isomorphic. 
The following corollary to Deligne's lemma will be used in the next section to classify tensor ideals in $\underline{\operatorname{Rep}}\left(S_{d}\right)$.

Corollary 3.12. Every nonzero tensor ideal in $\underline{\operatorname{Rep}}\left(S_{d}\right)$ contains a nonzero identity morphism.

Proof. Suppose $\mathscr{I}$ is a nonzero tensor ideal in $\operatorname{Rep}\left(S_{d}\right)$. Since tensor ideals are closed under composition, it suffices to show that $\mathscr{I}$ contains a morphism which has a nonzero isomorphism as a direct summand. Let $f$ be a nonzero morphism in $\mathscr{\Phi}$. Then, by Remark $2.5, \mathrm{id}_{\Delta} \otimes f$ is also a nonzero morphism in $\Phi$. By Lemma 3.11, we have $\operatorname{id}_{\Delta} \otimes f=\mathscr{F}_{\Delta}\left(f^{\prime}\right)$ for some nonzero morphism $f^{\prime}$ in $\underline{\operatorname{Rep}}\left(S_{-1}\right)$. Since $\underline{\operatorname{Rep}}\left(S_{-1}\right)$ is semisimple (see [Deligne 2007, Théorème 2.18] or [Comes and Ostrik 2011, Corollary 5.21]) it follows that $f^{\prime}$ (and therefore $\mathscr{F}_{\Delta}\left(f^{\prime}\right)$ ) is the direct sum of isomorphisms and zero morphisms.

3E. Tensor ideals in $\underline{\operatorname{Rep}}\left(S_{d}\right)$. In this section we use results from [Comes and Ostrik 2011] along with Corollary 3.12 to classify tensor ideals in $\underline{\operatorname{Rep}}\left(S_{d}\right)$ for arbitrary $d \in \mathbb{Z}_{\geq 0}{ }^{8}$ We begin by introducing an equivalence class on Young diagrams:

Definition 3.13. Consider the weakest equivalence relation on the set of all Young diagrams such that $\lambda$ and $\mu$ are equivalent whenever the indecomposable object $L(\lambda)$ is a direct summand of $L(\mu) \otimes[\mathrm{pt}]$ in $\operatorname{Rep}\left(S_{d}\right)$. When $\lambda$ and $\mu$ are in the same equivalence class we write $\lambda \stackrel{d}{\approx} \mu$.

The following proposition contains enough information on the equivalence relation $\stackrel{d}{\approx}$ for us to classify tensor ideals in $\underline{\operatorname{Rep}}\left(S_{d}\right)$.

Proposition 3.14. Assume $d$ is a nonnegative integer and $\lambda$, $\mu$ are Young diagrams.

(1) A nonzero morphism of the form $L(\lambda) \rightarrow L(\mu)$ is a negligible morphism in $\operatorname{Rep}\left(S_{d}\right)$ if and only if $L(\lambda)$ or $L(\mu)$ is not the minimal indecomposable object in an infinite block of $\underline{\operatorname{Rep}}\left(S_{d}\right)$.

(2) $\lambda \stackrel{d}{\approx} \mu$ whenever $L(\lambda)$ and $L(\mu)$ are in trivial blocks of $\underline{\operatorname{Rep}}\left(S_{d}\right)$.

(3) $\lambda \stackrel{d}{\approx} \mu$ whenever $L(\lambda)$ is a nonminimal indecomposable object in an infinite block and $L(\mu)$ is in a trivial block of $\underline{\operatorname{Rep}}\left(S_{d}\right)$.

(4) $\lambda \stackrel{d}{\approx} \mu$ whenever neither $L(\lambda)$ nor $L(\mu)$ is a minimal indecomposable object in an infinite block of $\underline{\operatorname{Rep}}\left(S_{d}\right)$.

(5) Suppose $\lambda \stackrel{d}{\approx} \mu$ and $\Phi$ is a tensor ideal in $\underline{\operatorname{Rep}}\left(S_{d}\right)$ containing $\operatorname{id}_{L(\lambda)}$. Then $\mathrm{id}_{L(\mu)}$ is also in $\Phi$.

${ }^{8}$ If $t \notin \mathbb{Z}_{\geq 0}$, then $\underline{\operatorname{Rep}}\left(S_{t}\right)$ is semisimple (see [Deligne 2007, Théorème 2.18] or [Comes and Ostrik 2011, Corollary 5.21]). Hence, there are no nonzero proper tensor ideals in $\underline{\operatorname{Rep}}\left(S_{t}\right)$ when $t \notin \mathbb{Z} \geq 0$. 
Proof. Part (1) follows from [Comes and Ostrik 2011, Proposition 3.25, Corollary 5.9, and Theorem 6.10]. Part (2) is easy to check using [Comes and Ostrik 2011, Propositions 3.12, 5.15 and Lemma 5.20(1)]. Part (4) follows from parts (2) and (3). Part (5) is easy to check. Hence, it suffices to prove part (3). To do so, let b denote the infinite block of $\underline{\operatorname{Rep}}\left(S_{d}\right)$ containing $L(\lambda)$. We will proceed by induction on $b$ with respect to $\prec$ (see [Comes and Ostrik 2011, Definition 5.12]).

If $\mathrm{b}$ is the minimal with respect to $\prec$, then using [Comes and Ostrik 2011, Proposition 3.12 and Lemmas 5.18(1) and 5.20(1)] we can find a Young diagram $\rho$ with $L(\rho)$ in a trivial block of $\underline{\operatorname{Rep}}\left(S_{d}\right)$ such that $\lambda \stackrel{d}{\approx} \rho$. By part $(2), \rho \stackrel{d}{\approx} \mu$ and we are done. Now suppose $b$ is not minimal with respect to $\prec$. Then, using [Comes and Ostrik 2011, Proposition 3.12 and Lemmas 5.18(2) and 5.20(2)], we can find a Young diagram $\rho^{\prime}$ with $\lambda \stackrel{d}{\approx} \rho^{\prime}$ such that $L\left(\rho^{\prime}\right)$ is in an infinite block b' of $\underline{\operatorname{Rep}}\left(S_{d}\right)$ with $\mathrm{b}^{\prime} \supsetneqq \mathrm{b}$. By induction $\rho^{\prime} \stackrel{d}{\approx} \mu$ and we are done.

We are now ready to classify tensor ideals in $\underline{\operatorname{Rep}}\left(S_{d}\right)$.

Theorem 3.15. If $d$ is a nonnegative integer, then the only nonzero proper tensor ideal in $\operatorname{Rep}\left(S_{d}\right)$ is the ideal of negligible morphisms.

Proof. Assume $\mathscr{I}$ is a nonzero proper tensor ideal of $\operatorname{Rep}\left(S_{d}\right)$. Then $\mathscr{I}$ is contained in the ideal of negligible morphisms (see [Freedman 2003, Proposition 3.1]), hence we must show that $\mathscr{I}$ contains all negligible morphisms. Suppose $\lambda$ is a Young diagram such that $L(\lambda)$ is not the minimal indecomposable object in an infinite block of $\underline{\operatorname{Rep}}\left(S_{d}\right)$. By Proposition 3.14(1), it suffices to show $\operatorname{id}_{L(\lambda)}$ is contained in $\mathscr{I}$. By Corollary 3.12, there exists a nonzero identity morphism in $\mathscr{I}$. It follows that $\mathscr{I}$ contains $\operatorname{id}_{L(\mu)}$ for some Young diagram $\mu$. In particular, $\operatorname{id}_{L(\mu)}$ is a negligible morphism. Hence, by Proposition 3.14(1), $L(\mu)$ is not the minimal indecomposable object in an infinite block of $\underline{\operatorname{Rep}}\left(S_{d}\right)$. Thus, by Proposition 3.14(4), $\lambda \stackrel{d}{\approx} \mu$. Finally, by Proposition 3.14(5), $\mathrm{id}_{L(\lambda)}$ is contained in $\mathscr{I}$.

Corollary 3.16. The tensor ideal in $\underline{\operatorname{Rep}}\left(S_{d}\right)$ generated by $\mathrm{id}_{\Delta}$ is the ideal of all negligible morphisms.

Proof. $\operatorname{id}_{\Delta}=x_{d+1}$ is a nonzero negligible morphism in $\underline{\operatorname{Rep}}\left(S_{d}\right)$ (see [Comes and Ostrik 2011, Remark 3.22]). Hence, the result follows from Theorem 3.15.

\section{The $t$-structure on $K^{b}\left(\underline{\operatorname{Rep}}\left(S_{d}\right)\right)$}

4A. Homotopy category. Let $\mathscr{A}$ be an additive category. Let $K^{b}(\mathscr{A})$ be the bounded homotopy category of $\mathscr{A}$; see, e.g., [Kashiwara and Schapira 2006, §11]. Thus the objects of $K^{b}(\mathscr{A})$ are finite complexes of objects in $\mathscr{A}$ and the morphisms are morphisms of complexes up to homotopy. The category $K^{b}(\mathscr{A})$ has a natural structure of a triangulated category; see loc. cit. In particular, for each integer $n$ we have a translation functor $[n]: K^{b}(\mathscr{A}) \rightarrow K^{b}(\mathscr{A})$. 
Any object $A \in \mathscr{A}$ can be considered as a complex $A[0]$ concentrated in degree 0 or, more generally, as a complex $A[n]$ concentrated in degree $-n$. Thus we have a fully faithful functor $\mathscr{A} \rightarrow K^{b}(\mathscr{A}), A \mapsto A[0]$. We will say that an object $K \in K^{b}(\mathscr{A})$ is split if it is isomorphic to an object of the form $\bigoplus_{i} A_{i}\left[n_{i}\right]$ with $A_{i} \in \mathscr{A}, n_{i} \in \mathbb{Z}$.

Now assume that $\mathscr{A}$ is an additive tensor category. The category $K^{b}(\mathscr{A})$ has a natural structure of an additive tensor category. If the category $\mathscr{A}$ is braided or symmetric then so is the category $K^{b}(\mathscr{A})$. The functor $\mathscr{A} \rightarrow K^{b}(\mathscr{A}), A \mapsto A[0]$ has an obvious structure of a (braided) tensor functor. If the category $\mathscr{A}$ is rigid so is the category $K^{b}(\mathscr{A})$.

4B. Definition of $\boldsymbol{t}$-structure. We can apply the construction from Section 4A to the case $\mathscr{A}=\underline{\operatorname{Rep}}\left(S_{d}\right)$. We obtain a triangulated tensor category $\mathscr{K}_{d}:=K^{b}\left(\underline{\operatorname{Rep}}\left(S_{d}\right)\right)$.

Proposition 4.1. For any $K \in \mathscr{K}_{d}$ the object $\Delta \otimes K$ is split.

Proof. By Lemma 3.11, the functor $\Delta \otimes-: \underline{\operatorname{Rep}}\left(S_{d}\right) \rightarrow \underline{\operatorname{Rep}}\left(S_{d}\right)$ is naturally isomorphic to a composition $\operatorname{Rep}\left(S_{d}\right) \rightarrow \underline{\operatorname{Rep}}\left(S_{-1}\right) \rightarrow \underline{\operatorname{Rep}}\left(S_{d}\right)$. The category $\underline{\operatorname{Rep}}\left(S_{-1}\right)$ is semisimple ([Deligne 2007, Théorème 2.18] or [Comes and Ostrik 2011, Corollary 5.21]), so every object of $K^{b}\left(\underline{\operatorname{Rep}}\left(S_{-1}\right)\right)$ is split. The result follows.

We define $\mathscr{K}_{d}^{\leq 0}$ as the full subcategory of $\mathscr{K}_{d}$ consisting of objects $K$ such that $\Delta \otimes K$ is concentrated in nonpositive degrees (that is, isomorphic to $\bigoplus_{i} A_{i}\left[n_{i}\right]$ with $A_{i} \in \mathscr{A}$ and $n_{i} \in \mathbb{Z}_{\geq 0}$ ). Similarly, we define $\mathscr{K}_{d}^{\geq 0}$ as the full subcategory of $\mathscr{K}_{d}$ consisting of objects $K$ such that $\Delta \otimes K$ is concentrated in nonnegative degrees. The following result will be proved in Section $4 \mathrm{C}$.

Theorem 4.2. The pair $\left(\mathscr{K}_{\frac{d}{d}}^{\leq 0}, \mathscr{K}_{\frac{d}{}}^{\geq 0}\right)$ is a t-structure (see [Beبllinson et al. 1982, Définition 1.3.1]) on the category $\mathscr{K}_{d}$.

Recall that the core of this $t$-structure is the subcategory $\mathscr{K}_{d}^{0}=\mathscr{K}_{\frac{d}{d}}^{\leq 0} \cap K_{\frac{d}{2}}^{\geq 0}$. By definition this means that $K \in \mathscr{K}_{d}^{0}$ if and only if $\Delta \otimes K$ is concentrated in degree zero. In particular, for any $A \in \underline{\operatorname{Rep}}\left(S_{d}\right)$ the object $A[0]$ is in $\mathcal{K}_{d}^{0}$.

Corollary 4.3. (a) The category $\mathcal{K}_{d}^{0}$ is abelian.

(b) The category $\mathscr{K}_{d}^{0}$ is a tensor subcategory of $\mathscr{K}_{d}$.

Proof. Part (a) follows from Theorem 4.2 and [Beǔlinson et al. 1982, Théorème 1.3.6]. For (b) we need to check that for $K, K^{\prime} \in \mathscr{K}_{d}^{0}$ we have $K \otimes K^{\prime} \in \mathscr{K}_{d}^{0}$. Assume this is not the case. This means that the split complex $\Delta \otimes K \otimes K^{\prime}$ is not concentrated in degree zero. Since $\Delta \otimes X \neq 0$ for any $0 \neq X \in \underline{\operatorname{Rep}}\left(S_{d}\right)$ (see Remark 2.5) we get that $\Delta \otimes \Delta \otimes K \otimes K^{\prime}$ is split and not concentrated in degree zero. But this is not the case since $\Delta \otimes \Delta \otimes K \otimes K^{\prime} \simeq(\Delta \otimes K) \otimes\left(\Delta \otimes K^{\prime}\right)$ and both $\Delta \otimes K$ and $\Delta \otimes K^{\prime}$ are split and concentrated in degree zero. 
We will show in Section $4 \mathrm{C}$ that the category $\mathscr{K}_{d}^{0}$ is actually pre-Tannakian. Thus we constructed a fully faithful tensor functor $\operatorname{Rep}\left(S_{d}\right) \rightarrow \mathscr{K}_{d}^{0}$, where $\mathscr{K}_{d}^{0}$ is a pre-Tannakian category. Of course a priori this might be quite different from Deligne's functor $\underline{\operatorname{Rep}}\left(S_{d}\right) \rightarrow \underline{\operatorname{Rep}}^{a b}\left(S_{d}\right)$.

4C. Verification of t-structure axioms. The main goal of this section is to prove Theorem 4.2.

4C1. We start by reformulating the definition of $\mathscr{K}_{d}^{\leq 0}$ and $\mathscr{K}_{\frac{d}{}}^{\geq 0}$ in terms of negligible objects, i.e., objects whose identity morphisms are negligible.

Proposition 4.4. Let $K \in \mathscr{K}_{d}$. Then $K \in \mathscr{K}_{\frac{1}{d}}^{\leq 0}$ if and only if $\operatorname{Hom}(K, A[n])=0$ for any negligible $A \in \underline{\operatorname{Rep}}\left(S_{d}\right)$ and $n \in \mathbb{Z}_{<0}$. Similarly, $K \in \mathscr{K}_{\bar{d}}^{\geq 0}$ if and only if $\operatorname{Hom}(K, A[n])=0$ for any negligible $A \in \underline{\operatorname{Rep}}\left(S_{d}\right)$ and $n \in \mathbb{Z}_{>0}$.

Proof. We prove only the characterization of $K_{d}^{\leq 0}$ (the case of $\mathscr{K}_{\frac{d}{d}}^{\geq 0}$ is similar). Assume first that $\operatorname{Hom}(K, A[n])=0$ for any negligible $A$ and $n \in \mathbb{Z}_{<0}$. By Proposition 3.2 $\Delta^{*}=\Delta$, thus by Corollary $3.16 \Delta \otimes B=\Delta^{*} \otimes B$ is negligible for all $B \in \underline{\operatorname{Rep}}\left(S_{d}\right)$. Hence, $\operatorname{Hom}(\Delta \otimes K, B[n])=\operatorname{Hom}\left(K, \Delta^{*} \otimes B[n]\right)=0$ for any $B \in \underline{\operatorname{Rep}}\left(S_{d}\right)$ and $n \in \mathbb{Z}_{<0}$. Since by Proposition 4.1 the object $\Delta \otimes K \in \mathscr{K}_{d}$ is split we get immediately that $K \in \mathscr{K}_{d}^{\leq 0}$.

Conversely, assume that $K \in \mathscr{K}_{d}^{\leq 0}$. Then by definition $\operatorname{Hom}(\Delta \otimes K, B[n])=0$ for any $B \in \underline{\operatorname{Rep}}\left(S_{d}\right)$ and $n \in \mathbb{Z}_{<0}$. Hence $\operatorname{Hom}\left(K, \Delta^{*} \otimes B[n]\right)=0$. Since, by Corollary 3.16, any negligible object is a direct summand of an object of the form $\Delta \otimes B=\Delta^{*} \otimes B$ we are done.

4C2. Blockwise description of $\left(\mathscr{K}_{d}^{\leq 0}, \Re_{d}^{\geq 0}\right)$. Recall that the category $\underline{\operatorname{Rep}}\left(S_{d}\right)$ decomposes into a direct sum of blocks $\underline{\operatorname{Rep}}\left(S_{d}\right)=\bigoplus_{\mathrm{b}} \underline{\operatorname{Rep}}\left(S_{d}\right)$; see Section 2B4. Similarly, we have a decomposition $\mathscr{K}_{d}=\bigoplus_{\mathrm{b}}\left(\mathscr{K}_{d}\right)_{\mathrm{b}}$ (in other words, for any object $K \in \mathscr{K}_{d}$ we have a canonical decomposition $K=\bigoplus_{\mathrm{b}} K_{\mathrm{b}}$ where all the terms of the complex $K_{\mathrm{b}} \in\left(\mathscr{K}_{d}\right)_{\mathrm{b}}$ are in the block $\left.\underline{\operatorname{Rep}}_{\mathrm{b}}\left(S_{d}\right)\right)$. Since $\Delta \otimes\left(\bigoplus_{\mathrm{b}} K_{\mathrm{b}}\right)=\bigoplus_{\mathrm{b}} \Delta \otimes K_{\mathrm{b}}$ we see that $K=\bigoplus_{\mathrm{b}} K_{\mathrm{b}} \in \mathscr{K}_{d}^{\leq 0}$ if and only if $K_{\mathrm{b}} \in \mathscr{K}_{d}^{\leq 0}$ for any b (and similarly for $\left.\mathscr{K}_{\frac{d}{d}}^{\geq 0}\right)$. In other words $\mathscr{K}_{d}^{\leq 0}=\bigoplus_{\mathrm{b}}\left(\mathscr{K}_{\frac{1}{d}}^{\leq 0}\right)_{\mathrm{b}}$ where $\left(\mathscr{K}_{d}^{\leq 0}\right)_{\mathrm{b}}=\mathscr{K}_{\frac{d}{d}}^{\leq 0} \cap\left(\mathscr{K}_{d}\right)_{\mathrm{b}}$, that is, the subcategory $\mathscr{K}_{d}^{\leq 0}$ is compatible with the block decomposition (and similarly for $\left.\mathscr{K}_{d}^{\geq 0}=\bigoplus_{\mathrm{b}}\left(\mathscr{K}_{d}^{\geq 0}\right)_{\mathrm{b}}\right)$. Thus in order to verify that $\left(\mathscr{K}_{d}^{\leq 0}, \mathscr{K}_{d}^{\geq 0}\right)$ is a $t$-structure on $\mathscr{K}_{d}$ it is sufficient to verify that $\left(\left(\mathscr{K}_{d}^{\leq 0}\right)_{\mathrm{b}},\left(\mathscr{K}_{d}^{\geq 0}\right)_{\mathrm{b}}\right)$ is a $t$-structure on $\left(\mathscr{K}_{d}\right)_{\mathrm{b}}$ for every block b. Fortunately, Proposition 4.4 gives rise to an easy description of $\left(\mathscr{K}_{\frac{d}{d}}^{\leq 0}\right)_{\mathrm{b}}$ and $\left(\mathcal{K}_{\bar{d}}^{\geq 0}\right)_{\mathrm{b}}$.

Proposition 4.5. Let $K \in\left(\mathscr{K}_{d}\right)_{\mathrm{b}}$.

(a) Assume that $\mathrm{b}$ is a semisimple block and let $L$ be a unique indecomposable object in $\mathrm{b}$. Then $K \in\left(\mathscr{K}_{d}^{\leq 0}\right)_{\mathrm{b}}$ (resp. $\left.K \in\left(\mathscr{K}_{d}^{\geq 0}\right)_{\mathrm{b}}\right)$ if and only if $K \in\left(\mathscr{K}_{d}\right)_{\mathrm{b}}$ and $\operatorname{Hom}(K, L[n])=0$ for any $n \in \mathbb{Z}_{<0}\left(\right.$ resp. for $\left.n \in \mathbb{Z}_{>0}\right)$. 
(b) Assume that $\mathrm{b}$ is a nonsemisimple block with indecomposable objects $L_{i}$ for $i \in \mathbb{Z}_{\geq 0}$ labeled as in Proposition 2.7(ii). Then $K \in\left(\mathcal{K}_{\frac{d}{d}}^{\leq 0}\right)_{\mathrm{b}}\left(\right.$ resp. $\left.K \in\left(\mathcal{K}_{\bar{d}}^{\geq 0}\right)_{\mathrm{b}}\right)$ if and only if $K \in\left(\mathscr{K}_{d}\right)_{\mathrm{b}}$ and $\operatorname{Hom}\left(K, L_{i}[n]\right)=0$ for all $i>0$ and any $n \in \mathbb{Z}_{<0}$ (resp. for $n \in \mathbb{Z}_{>0}$ ).

Proof. Combine Proposition 4.4 and Proposition 2.7.

4C3. Analogy with Temperley-Lieb category. The definition of the $t$-structure in Section $4 \mathrm{~B}$ was motivated by the following analogy. Pick a nontrivial root of unity $q$ such that $q+q^{-1} \in F$ and recall the Temperley-Lieb category $T L(q)$ from Section 2C. Consider the category $K^{b}(T L(q))$. It is well known (see, e.g., [Ostrik 2008, Proposition 2.7] that the embedding $T L(q) \subset \mathscr{C}_{q}$ induces an equivalence of triangulated categories $K^{b}(T L(q)) \simeq D^{b}\left(\mathscr{C}_{q}\right)$, where $D^{b}\left(\mathscr{C}_{q}\right)$ is the derived category of the abelian category $\mathscr{C}_{q}$. In particular the category $\mathscr{D}_{q}:=K^{b}(T L(q))$ inherits a natural $t$-structure $\left(\mathscr{D}_{q}^{\leq 0}, \mathscr{D}_{q}^{\geq 0}\right)$ from the category $D^{b}\left(\mathscr{C}_{q}\right)$; see, e.g., [Berllinson et al. 1982, Exemple 1.3.2(i)]. ${ }^{9}$ This $t$-structure can be characterized as follows.

Let $\mathrm{St}:=V_{l-1} \in T L(q)$ be the so-called Steinberg module. It is known (see [Andersen et al. 1991, Theorem 9.8]) that $\mathrm{St}$ is a projective object of the category $\mathscr{C}_{q}$. Thus $\mathrm{St} \otimes Y$ is a projective object of $\mathscr{C}_{q}$ for any $Y \in \mathscr{C}_{q}$; see [Andersen et al. 1991, Lemma 9.10]. In particular, for any $K \in \mathscr{D}_{q}$ the object $\mathrm{St} \otimes K \in \mathscr{D}_{q}$ is isomorphic to its cohomology (as a finite complex consisting of projective modules and with projective cohomology). It is well known that each projective object of $\mathscr{C}_{q}$ is contained in $T L(q) \subset \mathscr{C}_{q}$; see [Andersen 1992, (5.7)]. Thus, in the language of Section 4A, for any $K \in K^{b}(T L(q))$ the complex St $\otimes K$ is split (analogous to Proposition 4.1). It is clear that $K \in \mathscr{D} \frac{\leq 0}{q}$ if and only if $\mathrm{St} \otimes K$ is concentrated in nonpositive degrees and similarly for $\mathscr{D}_{q}^{\geq 0}$. This is a counterpart of the definition of the $t$-structure $\left(K_{\frac{d}{d}}^{\leq 0}, \mathscr{K}_{\frac{d}{d}}^{\geq 0}\right)$.

Furthermore, it is known that each direct summand of $\mathrm{St} \otimes Y$ for $Y \in T L(q)$ is negligible (see [Andersen 1992, Proposition 3.5 and Lemma 3.6]) and that each negligible object of $T L(q)$ is a direct summand of $\operatorname{St} \otimes Y$ with $Y \in T L(q)$ (see [Andersen 1992, p. 158]). Thus we have the following counterpart of Proposition 4.4 (with a similar proof):

(a) Let $K \in \mathscr{D}_{q}$. Then $K \in \mathscr{D}_{q}^{\leq 0}$ (resp. $K \in \mathscr{D}_{q}^{\geq 0}$ ) if and only if $\operatorname{Hom}(K, A[n])=0$ for any negligible $A \in T L(q)$ and $n \in \mathbb{Z}_{<0}$ (resp. $n \in \mathbb{Z}_{>0}$ ).

Hence, following Section $4 \mathrm{C} 2$, we can give a blockwise description of the $t$-structure $\left(\mathscr{D}_{q}^{\leq 0}, \mathscr{D}_{q}^{\geq 0}\right)$. For a block b let $\left(\mathscr{D}_{q}\right)_{\mathrm{b}}$ denote the full subcategory of $\mathscr{D}_{q}=K^{b}(T L(q))$ consisting of complexes with all terms from the block b. Using Lemma 2.11 we obtain the following counterpart of Proposition 4.5:

${ }^{9}$ Thus the category $\mathscr{D}_{q} \leq 0$ consists of objects of $D^{b}\left(\mathscr{C}_{q}\right)$ with nontrivial cohomology only in nonpositive degrees and similarly for $\mathscr{D}_{\bar{q}}^{\geq 0}$. 
(b) Let $\mathrm{b}$ be a nonsemisimple block of $T L(q)$ with indecomposable objects $L_{i}$ for $i \in \mathbb{Z}_{\geq 0}$ labeled as in Remark 2.10. Let $K \in\left(\mathscr{D}_{q}\right)_{\mathrm{b}}$. Then $K \in \mathscr{D}_{q}^{\leq 0}$ (resp. $K \in \mathscr{D}_{\bar{q}}^{\geq 0}$ ) if and only if $\operatorname{Hom}\left(K, L_{i}[n]\right)=0$ for all $i>0$ and any $n \in \mathbb{Z}_{<0}\left(\right.$ resp. for $\left.n \in \mathbb{Z}_{>0}\right)$.

From this description it is clear that the pair $\left(\mathscr{D}_{q}^{\leq 0} \cap\left(\mathscr{D}_{q}\right)_{\mathrm{b}}, \mathscr{D}_{q}^{\geq 0} \cap\left(\mathscr{D}_{q}\right)_{\mathrm{b}}\right)$ of subcategories of $\left(\mathscr{D}_{q}\right)_{\mathrm{b}}$ corresponds to the pair $\left(\left(\mathscr{K}_{d}^{\leq 0}\right)_{\mathrm{b}^{\prime}},\left(\mathscr{K}_{\bar{d}}^{\geq 0}\right)_{\mathrm{b}^{\prime}}\right)$ under the equivalence $\left(\mathscr{D}_{q}\right)_{\mathrm{b}} \simeq\left(\mathscr{K}_{d}\right)_{\mathrm{b}^{\prime}}$ induced by the equivalence of blocks from Proposition 2.9. Since $\left(\mathscr{D}_{q}^{\leq 0} \cap\left(\mathscr{D}_{q}\right)_{\mathrm{b}}, \mathscr{D}_{q}^{\geq 0} \cap\left(\mathscr{D}_{q}\right)_{\mathrm{b}}\right)$ is a $t$-structure on the category $\left(\mathscr{D}_{q}\right)_{\mathrm{b}}$ we have the following:

Corollary 4.6. Let $\mathrm{b}$ be a nonsemisimple block of the category $T L(q)$ and let $\mathrm{b}^{\prime}$ be an equivalent block in the category $\underline{\operatorname{Rep}}\left(S_{d}\right)$ as in Proposition 2.9. Then $\left(\left(\mathscr{K}_{d}^{\leq 0}\right)_{\mathrm{b}^{\prime}},\left(\mathscr{K}_{d}^{\geq 0}\right)_{\mathrm{b}^{\prime}}\right)$ is a $t$-structure on the category $\left(\mathscr{K}_{d}\right)_{\mathrm{b}^{\prime}}$.

4C4. Proof of Theorem 4.2. It suffices to show $\left(\left(\mathscr{K}_{d}^{\leq 0}\right)_{\mathrm{b}},\left(\mathscr{K}_{d}^{\geq 0}\right)_{\mathrm{b}}\right)$ is a $t$-structure on $\left(\mathscr{K}_{d}\right)_{\mathrm{b}}$ for every block $\mathrm{b}$. If the block $\mathrm{b}$ is semisimple then the category $\left(\mathscr{K}_{d}\right)_{\mathrm{b}}$ can be identified with $K^{b}\left(\operatorname{Vec}_{F}\right)$ and Proposition $4.5(\mathrm{a})$ shows that $\left(\left(\mathcal{K}_{\frac{d}{d}}^{\leq 0}\right)_{\mathrm{b}},\left(\mathscr{K}_{d}^{\geq 0}\right)_{\mathrm{b}}\right)$ is the standard $t$-structure on $K^{b}\left(\operatorname{Vec}_{F}\right)$.

It remains to consider the case when $\mathrm{b}$ is a nonsemisimple block. Choose a nontrivial root of unity $q$ such that $q+q^{-1} \in F$ (for example, a primitive cubic root of unity $\zeta$ will work for any $F$ since $\zeta+\zeta^{-1}=-1 \in F$ ). Then there is a nonsemisimple block in $T L(q)$ which is equivalent to b (Proposition 2.9). Hence, by Corollary 4.6, $\left(\left(\mathscr{K}_{d}^{\leq 0}\right)_{\mathrm{b}},\left(\mathscr{K}_{d}^{\geq 0}\right)_{\mathrm{b}}\right)$ is a $t$-structure on $\left(\mathscr{K}_{d}\right)_{\mathrm{b}}$.

4C5. Complements. The proof in Section $4 \mathrm{C} 4$ implies the following:

Corollary 4.7. (a) The category $\mathscr{K}_{d}^{0}$ is pre-Tannakian.

(b) Any object of the category $\mathscr{K}_{d}^{0}$ is isomorphic to a subquotient of a direct sum of tensor powers of $[\mathrm{pt}]$.

Proof. We already know that the category $\mathscr{K}_{d}^{0}$ is an abelian tensor category (see Corollary 4.3). It is obvious that Hom's are finite-dimensional and $\operatorname{End}(\mathbf{1})=F$ since this is true in the category $\mathscr{K}_{d}$. The category $\mathscr{K}_{d}^{0}$ is rigid: if $\Delta \otimes K$ is concentrated in degree zero then the same is true for $\Delta \otimes K^{*} \simeq(\Delta \otimes K)^{*}$. It remains to check that any object of $\mathscr{K}_{d}^{0}$ has finite length. It is clear that we can verify this block by block. The result is clear for semisimple blocks since by Proposition 4.5(a) the core of the corresponding $t$-structure identifies with $\operatorname{Vec}_{F}$. This is also clear for nonsemisimple blocks since the corresponding $t$-structure (described in Proposition 4.5) identifies with the $t$-structure on a block of the Temperley-Lieb category and the corresponding core has all objects of finite length since this is true for the category $\mathscr{C}_{q}$. This proves (a).

For (b) we use the same argument as above: it is sufficient to verify the statement block by block. Here the result is trivial for semisimple blocks and is known for nonsemisimple ones since it is known to hold for the category $\mathscr{C}_{q}$. 
Remark 4.8. Using similar techniques of importing known results about the category $\mathscr{C}_{q}$ to the category $\mathscr{K}_{d}^{0}$ we can obtain detailed information about this category. In particular, we see that the category $\mathscr{K}_{d}^{0}$ has enough projective objects; all indecomposable projective objects are direct summands of tensor powers of [pt] (but powers of $[\mathrm{pt}]$ are not projective in general; for example $[\mathrm{pt}]^{\otimes 0}=\mathbf{1}$ is not projective). Thus Corollary 4.7(b) can be improved: any object of the category $\mathscr{K}_{d}^{0}$ is isomorphic to a quotient of a direct sum of tensor powers of [pt].

\section{Universal property}

\section{A. Extension property of the category $\Re_{d}^{0}$.}

Proposition 5.1. Let $\mathcal{T}$ be a pre-Tannakian category and let $\mathscr{F}: \underline{\operatorname{Rep}}\left(S_{d}\right) \rightarrow \mathscr{T}$ be a tensor functor. Assume that $\mathscr{F}(\Delta) \neq 0$. Then the functor $\mathscr{F}$ (uniquely) factorizes as $\underline{\operatorname{Rep}}\left(S_{d}\right) \rightarrow \mathscr{K}_{d}^{0} \rightarrow \mathcal{T}$, where $\mathscr{K}_{d}^{0} \rightarrow \mathcal{T}$ is an exact tensor functor.

Proof. Let $K \in \mathscr{K}_{d}^{0}$. We can consider $\mathscr{F}(K) \in K^{b}(\mathscr{T})$. Since the category $\mathscr{T}$ is abelian we can talk about cohomology of $\mathscr{F}(K)$.

Lemma 5.2. $H^{i}(\mathscr{F}(K))=0$ for $i \neq 0$.

Proof. Notice that for any $0 \neq X \in \mathscr{T}$ we have $X \otimes \mathscr{F}(\Delta) \neq 0$. Since the endofunctor $-\otimes \mathscr{F}(\Delta)$ of the category $\mathscr{T}$ is exact (see, e.g., [Bakalov and Kirillov 2001, Proposition 2.1.8]) we see that $H^{i}(\mathscr{F}(K \otimes \Delta))=H^{i}(\mathscr{F}(K) \otimes \mathscr{F}(\Delta))=H^{i}(\mathscr{F}(K)) \otimes \mathscr{F}(\Delta)$. By the definition of $\mathscr{K}_{d}^{0}$ the cohomology of $\mathscr{F}(K \otimes \Delta)$ is concentrated in degree zero and we are done.

We now define the functor $\mathscr{Y}_{d}^{0} \rightarrow \mathscr{T}$ as $K \mapsto H^{0}(\mathscr{F}(K))$ with the tensor structure induced by the one on $\mathscr{F}$ (or rather its extension to $K^{b}\left(\underline{\operatorname{Rep}}\left(S_{d}\right)\right) \rightarrow \mathscr{K}^{b}(\mathscr{T})$ ).

Remark 5.3. Here is an example of a tensor functor between abelian rigid tensor categories which is not exact. Let $k$ be a field of characteristic 2 and consider the category $\operatorname{Rep}_{k}(\mathbb{Z} / 2 \mathbb{Z})$ of finite-dimensional $k$-representations of $\mathbb{Z} / 2 \mathbb{Z}$. This category has precisely 2 indecomposable objects: one is simple and 1-dimensional; the other is projective and has categorical dimension 0 . Thus the quotient of $\operatorname{Rep}_{k}(\mathbb{Z} / 2 \mathbb{Z})$ by the negligible morphisms is equivalent to the category $\operatorname{Vec}_{k}$ of finitedimensional vector spaces over $k$. Clearly the quotient functor $\operatorname{Rep}_{k}(\mathbb{Z} / 2 \mathbb{Z}) \rightarrow \operatorname{Vec}_{k}$ is not exact since it sends the projective object to zero. One can also construct a similar example over a field of characteristic zero using the representation category of the additive supergroup of a 1-dimensional odd space.

5B. Fundamental groups of $\mathscr{K}_{d}^{\mathbf{0}}$ and $\operatorname{Rep}\left(S_{d}\right)$. Let $\pi$ be the fundamental group of the pre-Tannakian category $\mathscr{K}_{d}^{0}$. The action of $\pi$ on $[\mathrm{pt}] \in \underline{\operatorname{Rep}}\left(S_{d}\right) \subset \mathscr{K}_{d}^{0}$ defines a homomorphism $\pi \rightarrow S_{\mathbf{I}}$ where $\mathbf{I}=\operatorname{Spec}([\mathrm{pt}])$.

Proposition 5.4. The homomorphism $\varepsilon: \pi \rightarrow S_{\mathrm{I}}$ is in fact an isomorphism. 
Proof. Since the object [pt] generates $\mathscr{K}_{d}^{0}$ (see Corollary 4.7(b)) the homomorphism $\varepsilon: \pi \rightarrow S_{\mathrm{I}}$ is an embedding.

Consider the category $\operatorname{Rep}\left(S_{\mathbf{I}}, \varepsilon\right)$. It is shown in (the proof of) [Deligne 2007, Proposition B1] that its fundamental group is precisely the group $S_{\mathbf{I}}^{\varepsilon}=\operatorname{Aut}_{\operatorname{Rep}\left(S_{\mathbf{I}}, \varepsilon\right)}(\mathbf{I})$. We have an obvious tensor functor $\operatorname{Rep}\left(S_{d}\right) \rightarrow \operatorname{Rep}\left(S_{\mathbf{I}}, \varepsilon\right)$; by Proposition 5.1 it extends to a tensor functor $\mathscr{F}_{\mathrm{F}}: \mathscr{K}_{d}^{0} \rightarrow \operatorname{Rep}\left(S_{\mathbf{I}}, \varepsilon\right)$. Thus we have a homomorphism $S_{\mathbf{I}}^{\varepsilon} \rightarrow \mathscr{F}(\pi)$. It is clear that the composition $S_{\mathbf{I}}^{\varepsilon} \rightarrow \mathscr{F}(\pi) \subset \mathscr{F}\left(S_{\mathbf{I}}\right)=S_{\mathbf{I}}^{\varepsilon}$ is the identity map. The result follows.

5C. Proof of Theorem 1.2. We start with the following result:

Proposition 5.5 [Deligne 1990, 8.14(ii)]. The fundamental group of the category $\operatorname{Rep}\left(S_{d}\right)$ is the group $S_{d}$ acting on itself by conjugation.

Remark 5.6. It is explained in loc. cit. that we can replace $S_{d}$ with any affine algebraic group $G$ in the statement of the proposition.

Theorem 5.7. Let $\mathcal{T}$ be a pre-Tannakian category and let $\mathscr{F}: \underline{\operatorname{Rep}}\left(S_{d}\right) \rightarrow \mathscr{T}$ be a tensor functor with $T=\mathscr{F}([\mathrm{pt}])$.

(a) If $\mathscr{F}_{(}(\Delta)=0$, then the category $\operatorname{Rep}\left(S_{\mathbf{I}}, \varepsilon\right)$ endowed with the functor $\mathscr{F}_{T}$ : $\underline{\operatorname{Rep}}\left(S_{d}\right) \rightarrow \operatorname{Rep}\left(S_{\mathbf{I}}, \varepsilon\right)$ is equivalent to $\operatorname{Rep}\left(S_{d}\right)$ equipped with the functor $\underline{\operatorname{Rep}}\left(S_{d}\right) \rightarrow \operatorname{Rep}\left(S_{d}\right)$.

(b) If $\mathscr{F}_{(}(\Delta) \neq 0$, then the category $\operatorname{Rep}\left(S_{\mathbf{I}}, \varepsilon\right)$ endowed with the functor $\mathscr{F}_{T}$ : $\underline{\operatorname{Rep}}\left(S_{d}\right) \rightarrow \operatorname{Rep}\left(S_{\mathbf{I}}, \varepsilon\right)$ is equivalent to $K_{d}^{0}$ equipped with the functor $\underline{\operatorname{Rep}}\left(S_{d}\right) \rightarrow$ $\mathcal{Y}_{d}^{0}$.

Proof. (a) In this case $\mathscr{F}$ factorizes as $\operatorname{Rep}\left(S_{d}\right) \rightarrow \operatorname{Rep}\left(S_{d}\right) \rightarrow \mathscr{T}$ (see Corollary 3.16). The result follows from [Deligne 1990, Théorème 8.17] and Proposition 5.5.

(b) In this case $\mathscr{F}$ extends to a functor $\operatorname{Rep}\left(S_{d}\right) \rightarrow \mathscr{K}_{d}^{0} \rightarrow \mathscr{T}$ by Proposition 5.1. The result follows from [Deligne 1990, Théorème 8.17] and Proposition 5.4.

If we apply Theorem $5.7(\mathrm{~b})$ to the category $\mathscr{T}=\underline{\operatorname{Rep}}\left(S_{-1}\right)$ and the functor $\underline{\operatorname{Res}}_{S_{-1}}^{S_{d}}: \underline{\operatorname{Rep}}\left(S_{d}\right) \rightarrow \mathcal{T}$ described in Definition 2.4 and Section 3D we obtain the following:

Corollary 5.8. The category $\underline{\operatorname{Rep}}^{a b}\left(S_{d}\right)$ endowed with the functor $\operatorname{Rep}\left(S_{d}\right) \rightarrow$ $\underline{\operatorname{Rep}}^{a b}\left(S_{d}\right)$ is equivalent to the category $\mathscr{K}_{d}^{0}$ with the functor $\underline{\operatorname{Rep}}\left(S_{d}\right) \rightarrow \mathscr{K}_{d}^{0}$.

Clearly Theorem 5.7 and Corollary 5.8 together imply Theorem 1.2.

\section{Acknowledgments}

This paper owes its existence to Pierre Deligne, who explained to the second author a proof of Lemma 3.11, which is crucial to this paper, during a visit to the Institute for Advanced Study. Both authors are happy to express their deep gratitude to him 
and to the Institute for Advanced Study, which made this interaction possible. The authors are also very grateful to Alexander Kleshchev, who initiated this project. We also thank Michael Finkelberg and Friedrich Knop for their interest in this work and Darij Grinberg for his detailed comments. The second author's work was partially supported by NSF grant DMS-0602263.

\section{References}

[Andersen 1992] H. H. Andersen, "Tensor products of quantized tilting modules", Comm. Math. Phys. 149:1 (1992), 149-159. MR 94b:17015 Zbl 0760.17004

[Andersen et al. 1991] H. H. Andersen, P. Polo, and K. X. Wen, "Representations of quantum algebras”, Invent. Math. 104:1 (1991), 1-59. MR 92e:17011 Zbl 0724.17012

[Bakalov and Kirillov 2001] B. Bakalov and A. Kirillov, Jr., Lectures on tensor categories and modular functors, University Lecture Series 21, Amer. Math. Soc., Providence, RI, 2001. MR 2002d:18003 Zbl 0965.18002

[Bě̆linson et al. 1982] A. A. Beǔlinson, J. Bernstein, and P. Deligne, "Faisceaux pervers", pp. 5-171 in Analysis and topology on singular spaces, I (Luminy, 1981)), Astérisque 100, Soc. Math. France, Paris, 1982. MR 86g:32015 Zbl 0536.14011

[Comes and Ostrik 2011] J. Comes and V. Ostrik, "On blocks of Deligne's category $\underline{\operatorname{Rep}}\left(S_{t}\right)$ ", $A d v$. Math. 226:2 (2011), 1331-1377. MR 2012b:20020 Zbl 1225.18005

[Deligne 1990] P. Deligne, "Catégories tannakiennes", pp. 111-195 in The Grothendieck Festschrift, II, edited by P. Cartier et al., Progr. Math. 87, Birkhäuser, Boston, 1990. MR 92d:14002 Zbl 0727.14010

[Deligne 2007] P. Deligne, "La catégorie des représentations du groupe symétrique $S_{t}$, lorsque $t$ n'est pas un entier naturel”, pp. 209-273 in Algebraic groups and homogeneous spaces, edited by V. B. Mehta, Tata Inst. Fund. Res., Mumbai, 2007. MR 2009b:20021 Zbl 1165.20300

[Freedman 2003] M. H. Freedman, "A magnetic model with a possible Chern-Simons phase", Comm. Math. Phys. 234:1 (2003), 129-183. MR 2004b:81193 Zbl 1060.81054

[Fulton and Harris 1991] W. Fulton and J. Harris, Representation theory: A first course, Graduate Texts in Mathematics 129, Springer, New York, 1991. MR 93a:20069 Zbl 0744.22001

[Kashiwara and Schapira 2006] M. Kashiwara and P. Schapira, Categories and sheaves, Grundlehren der Mathematischen Wissenschaften 332, Springer, Berlin, 2006. MR 2006k:18001 Zbl 1118.18001

[Ostrik 2008] V. Ostrik, "Module categories over representations of $\mathrm{SL}_{q}(2)$ in the non-semisimple case”, Geom. Funct. Anal. 17:6 (2008), 2005-2017. MR 2009a:20082 Zbl 1139.18007

Communicated by Susan Montgomery

Received 2013-04-11 Accepted 2013-08-31

jcomes@math.uoregon.edu

Department of Mathematics, University of Oregon, Eugene, OR 97403, United States

vostrik@math.uoregon.edu

Department of Mathematics, University of Oregon, Eugene, OR 97403, United States 


\section{Algebra \& Number Theory}

msp.org/ant

\section{EDITORS}

MANAGING EDITOR

Bjorn Poonen

Massachusetts Institute of Technology

Cambridge, USA

\author{
EDITORIAL BOARD CHAIR \\ David Eisenbud \\ University of California \\ Berkeley, USA
}

\section{BOARD OF EDITORS}

Georgia Benkart

Dave Benson

Richard E. Borcherds

John H. Coates

J-L. Colliot-Thélène

Brian D. Conrad

Hélène Esnault

Hubert Flenner

Edward Frenkel

Andrew Granville

Joseph Gubeladze

Roger Heath-Brown

Ehud Hrushovski

Craig Huneke

Mikhail Kapranov

Yujiro Kawamata

János Kollár

Yuri Manin

Barry Mazur

Philippe Michel
University of Wisconsin, Madison, USA

University of Aberdeen, Scotland

University of California, Berkeley, USA

University of Cambridge, UK

CNRS, Université Paris-Sud, France

University of Michigan, USA

Freie Universität Berlin, Germany

Ruhr-Universität, Germany

University of California, Berkeley, USA

Université de Montréal, Canada

San Francisco State University, USA

Oxford University, UK

Hebrew University, Israel

University of Virginia, USA

Yale University, USA

University of Tokyo, Japan

Princeton University, USA

Northwestern University, USA

Harvard University, USA

École Polytechnique Fédérale de Lausanne
Susan Montgomery

Shigefumi Mori

Raman Parimala

Jonathan Pila

Victor Reiner

Karl Rubin

Peter Sarnak

Joseph H. Silverman

Michael Singer

Vasudevan Srinivas

J. Toby Stafford

Bernd Sturmfels

Richard Taylor

Ravi Vakil

Michel van den Bergh

Marie-France Vignéras

Kei-Ichi Watanabe

Efim Zelmanov

Shou-Wu Zhang
University of Southern California, USA

RIMS, Kyoto University, Japan

Emory University, USA

University of Oxford, UK

University of Minnesota, USA

University of California, Irvine, USA

Princeton University, USA

Brown University, USA

North Carolina State University, USA

Tata Inst. of Fund. Research, India

University of Michigan, USA

University of California, Berkeley, USA

Harvard University, USA

Stanford University, USA

Hasselt University, Belgium

Université Paris VII, France

Nihon University, Japan

University of California, San Diego, USA

Princeton University, USA

PRODUCTION

production@msp.org

Silvio Levy, Scientific Editor

See inside back cover or msp.org/ant for submission instructions.

The subscription price for 2014 is US $\$ 225 /$ year for the electronic version, and $\$ 400 /$ year $(+\$ 55$, if shipping outside the US) for print and electronic. Subscriptions, requests for back issues and changes of subscribers address should be sent to MSP.

Algebra \& Number Theory (ISSN 1944-7833 electronic, 1937-0652 printed) at Mathematical Sciences Publishers, 798 Evans Hall \#3840, c/o University of California, Berkeley, CA 94720-3840 is published continuously online. Periodical rate postage paid at Berkeley, CA 94704, and additional mailing offices.

ANT peer review and production are managed by EditFLOW ${ }^{\circledR}$ from Mathematical Sciences Publishers.

\section{PUBLISHED BY}

- mathematical sciences publishers

nonprofit scientific publishing

http://msp.org/

(C) 2014 Mathematical Sciences Publishers 


\section{Algebra \& Number Theory}

Volume $8 \quad$ No. $2 \quad 2014$

Large self-injective rings and the generating hypothesis

LEIGH SHEPPERSON and NEIL STRICKLAND

On lower ramification subgroups and canonical subgroups

303

SHIN HATTORI

Wild models of curves

DINO LORENZINI

Geometry of Wachspress surfaces

COREY IRVING and HAL SCHENCK

Daniel Goldstein, Robert M. GuRAlnick, Mark L. Lewis, AleXander Moretó, Gabriel Navarro and Pham HuU TieP

The homotopy category of injectives

AMNON NEEMAN

Essential dimension of spinor and Clifford groups

Vladimir Chernousov and AleXANDER MERKurJeV

On Deligne's category $\underline{\operatorname{Rep}}^{a b}\left(S_{d}\right)$

JONATHAN COMES and VICTOR OSTRIK

Algebraicity of the zeta function associated to a matrix over a free group algebra

CHRISTIAN KASSEL and Christophe REUTENAUER 